\title{
Simulating Organizational Data from Redacted Input for Inference Enterprise Modeling
}

\author{
PAUL J. STICHA and TIRSO E. DIAZ, Human Resources Research Organization (HumRRO) \\ ELISE T. AXELRAD, SEAN D. VERMILLION, and DENNIS M. BUEDE, Innovative Decisions \\ International, an ITA International Company
}

\begin{abstract}
Organizations that use data to assess insider threats, or other workforce outcomes, need to evaluate the quality of their assessment methods. This evaluation relies on an accurate representation of the predictors and criterion variables within the organization's workforce. However, privacy concerns often limit the information that is available for evaluation. For example, the organization might anonymize identifying information of its workforce, or the evaluation might be restricted to use group statistics, such as marginal distributions of predictors and criteria, along with their intercorrelations. In this paper we demonstrate a hybrid approach for simulating workforce data to support inference-enterprise evaluation, including the crowdsourced elicitation of marginal distributions and correlations of predictors and the simulation of a workforce population from the statistical properties of a redacted set of predictor distributions. The methods provide a way to simulate a population that has statistical characteristics of the workforce, in order to assess the performance of the assessment methods. The statistical methods are supplemented by expert judgments for situations where required information is not available. We evaluate these methods using anonymized data from an actual organization.
\end{abstract}

CCS Concepts: • Security and privacy $\rightarrow$ Social aspects of security and privacy; $\bullet$ Computing methodologies $\rightarrow$ Simulation evaluation; Uncertainty quantification; • Mathematics of computing $\rightarrow$ Multivariate statistics;

Additional Key Words and Phrases: Inference enterprise modeling, insider threat identification, population simulation

ACM Reference format:

Paul J. Sticha, Tirso E. Diaz, Elise T. Axelrad, Sean D. Vermillion, and Dennis M. Buede. 2021. Simulating Organizational Data from Redacted Input for Inference Enterprise Modeling. Digit. Threat.: Res. Pract. 3, 1, Article 4 (October 2021), 30 pages. https://doi.org/10.1145/3457910

Paul J. Sticha is currently at PsychInference, LLC. Elise T. Axelrad is currently at Pacific Architects and Engineers. Sean D. Vermillion is currently at General Motors.

Research reported here was supported under Intelligence Advanced Research Projects Activity (IARPA) contract 2016-16031400006. The content is solely the responsibility of the authors and does not necessarily represent the official views of the U.S. Government.

Authors' addresses: P. J. Sticha, PsychInference, LLC, 8421 Sulky Ct., Alexandria VA 22308; email: paul.sticha@psychinference.com; T. E. Diaz, Human Resources Research Organization (HumRRO), 66 Canal Center Plaza, Suite 700, Alexandria VA 22314; email: tdiaz@humrro.org; E. T. Axelrad, Pacific Architects and Engineers, 7799 Leesburg Pike, Suite 300 North, Falls Church VA 22043; email: elise.axelrad@pae.com; S. D. Vermillion, GM IT Innovation Center, 13201 McCallen Pass, Austin TX 78753; email: sean.vermillion@gm.com; D. M. Buede, Innovative Decisions International, an ITA International Company, 1951 Kidwell Dr., Suite 750, Vienna VA 22182; email: dmbuede@innovativedecisions.com. Permission to make digital or hard copies of all or part of this work for personal or classroom use is granted without fee provided that copies are not made or distributed for profit or commercial advantage and that copies bear this notice and the full citation on the first page. Copyrights for components of this work owned by others than ACM must be honored. Abstracting with credit is permitted. To copy otherwise, or republish, to post on servers or to redistribute to lists, requires prior specific permission and/or a fee. Request permissions from permissions@acm.org.

(c) 2021 Association for Computing Machinery.

2576-5337/2021/10-ART4 \$15.00

https://doi.org/10.1145/3457910

Digital Threats: Research and Practice, Vol. 3, No. 1, Article 4. Publication date: October 2021. 


\section{INTRODUCTION}

Organizations frequently collect and analyze workforce data and information about the external environment to assess threats and opportunities that can affect their performance, and to predict the effects of policy changes on mission outcomes. Collection, analysis, and modeling of organizational and environmental data is an example of an Inference Enterprise (IE), which is defined as a unit within an organization that uses data, tools, people, and processes to make inferences about variables that are crucial for organizational success [Buede 2018]. An IE can focus on any of a wide variety of issues and can consider a comparably wide range of target variables (representing specific threats or opportunities) and associated predictive indicators.

Development and implementation of an IE requires a significant investment of time and money. Consequently, it is important for organizations to anticipate how well their IEs will perform to maximize the benefit from their investment. An Inference Enterprise Model (IEM) is a tool for forecasting the performance of an IE. For example, an IEM might be used to estimate how likely it is for an IE to correctly identify an existing threat or opportunity (termed recall), or how likely it is for the IE to identify a threat or opportunity when none exists (false positive rate). To evaluate the performance of an IE, the IEM must have information about (a) the joint distribution of target and indicator variables within the organization, (b) the methods that are used to aggregate the values of several indicator variables to estimate threat risk (e.g., logistic regression, Naïve Bayes nets), and (c) the criteria that are used to issue an alert when risk is high (e.g., what the probability of a threat should be for a case to be investigated further). An accurate representation of the joint distribution of relevant variables is a precondition for successful testing of aggregation methods and alert procedures, and consequently is the primary focus of this paper.

The research described in this paper developed and tested models of IEs that were primarily, but not exclusively, focused on assessing the risk of insider threats based on the data from a real, but unidentified, organization. The research was conducted as a sponsored competition among three contractor teams, who received quarterly challenge problems that described the target behaviors that were the focus of the IE, the methods used to assess the level of threat, and the criteria for alerting the organization about likely threats. Along with each IE description, the competitors received a summary of the organizational data regarding the relevant variables. Because of privacy concerns, we received no individual level data, but rather we got descriptive statistics, histograms, and correlations. Based on this information, we were asked to forecast the values for 15-60 conditional probabilities, including: (a) recall, or the proportion of individuals exhibiting the target behavior identified by the IE; (b) precision, or the proportion of individuals alerted by the IE who exhibit the target behavior; and (c) false positive rate, or the proportion of alerts among individuals who do not exhibit the target behavior. The sponsor, through their support contractor, assessed the accuracy of our forecasts.

Since target behaviors are often covert, an IE infers the likelihood of their existence from observable indicators. For example, the risk of espionage might be inferred from emails to foreign recipients, access to sensitive files, copying information to portable media, expressions of disgruntlement, foreign preference, and so forth. To support inferences, the IE collects information about employee behaviors and uses models to estimate the risk that an employee is engaging in espionage, conditional on the values of collected data [Buede 2018]. Thus, the focus on insider threat allows us to consider a range of target variables and indicators, such as the following:

- Predicting acts of sabotage, theft, or espionage by malicious employees or other insiders, based on personal characteristics, stressors, expectations, and troubling behaviors [Cappelli 2006; Greitzer 2011; Axelrad 2013];

- Predicting insider theft of intellectual property from their employer based on anomalous work behavior and personal associations [Moore 2011];

- Assessing the risks of other types of counterproductive work behavior based on personal characteristics and values [Mount 2006; Robinson 1995]; and

Digital Threats: Research and Practice, Vol. 3, No. 1, Article 4. Publication date: October 2021. 
- Inferring the likelihood of a cyber-attack based on the level and characteristics of network traffic [Cárdenas 2011].

Organizations that use live data streams to predict workforce outcomes are challenged by the imperfect predictability of human data, especially for rare outcomes, such as the presence of a nascent insider threat. If they engage consultants to evaluate the quality of such an IE, they introduce an extra data quality challenge when they obscure associations among predictors in the interest of protecting their employees' personally identifiable information. For example, organizations might anonymize personal information or completely redact this information, restricting the evaluator's access to distributional statistics, such as means, marginal distributions, and correlations. In this case, if one is to use powerful statistical modeling methods, it will be necessary to recover some of the individual distinctions from these aggregated data.

In this paper we demonstrate a hybrid approach for simulating the population of an organization to support IE evaluation, including the crowdsourced elicitation of marginal distributions and correlations of predictors and the simulated joint distribution of workforce population predictors and criteria, based on the statistical properties of a redacted set of predictor distributions. When the data were redacted so extensively that key statistical properties in employee data were missing, our imputation approach was to elicit statistical properties from behavioral experts. To assess the quality of this method for data simulation, we simulated a population for a problem for which we had received anonymized individual data after the competition was complete. We then compared the characteristics of the simulated population to the actual values provided from anonymized, individual, ground truth dataset. The quality of the forecasts of our IEMs has been described elsewhere by Buede [2018] and Brown [2019], so we will only summarize those results here.

We continue this paper by describing the context provided by the overall project for which this effort was a component. Then we provide a description of the methods developed to simulate a joint distribution of target behaviors and observed indicators, and to assess expert judgments of marginal distributions of and correlations between variables. We then describe the method that we used to assess the performance of both the simulation methods and the expert assessment procedures and present the results of that assessment. Finally, we summarize our results regarding the population simulation procedure, integrate them with our overall project results [Buede 2018] [Brown 2019], and discuss the implications of our results and avenues for additional research.

\section{CONTEXT FOR THE WORK}

In this section, we provide a summary of the overall project, based in part on the more detailed descriptions by Buede [2018] and Brown [2019]. This work was conducted in the context of the Scientific Advances to Continuous Insider Threat Evaluation (SCITE) Program, sponsored by the Intelligence Advanced Research Projects Activity (IARPA). The goal of the program was to improve the modeling and forecasting of performance of both existing and proposed insider threat detection enterprises. The program was structured as a competition among three contractor teams, who were given quarterly challenge problems, each problem containing 15-60 questions that required probabilistic forecasts consisting of a point estimate and a $60 \%$ certainty interval. The problem statements identified the target behavior, listed the indicators and detectors that were being considered, and described the methods that were being used to identify threats related to the target behavior. For example, here is an excerpt of the information provided for one of the challenge problems:

1. Target behavior: Makes data transfers using a work owned machine in patterns that are inconsistent with their peer group.

2. Indicators and detectors: Number of sent emails, average size of sent emails, number of emails larger than $5 \mathrm{MB}$, number of secure file transfer sessions where data were sent, average size of secure file transfer sessions where data were sent, and number of USB devices attached.

Digital Threats: Research and Practice, Vol. 3, No. 1, Article 4. Publication date: October 2021. 
3. Description of analyses (abbreviated and simplified): Use principal components analysis for each peer group. Then perform density-based spatial clustering of applications with noise (DBSCAN) to identify outliers and issue an alert for these outliers.

In addition, the problem statements posed the performance questions, which were stated as conditional probabilities to be forecasted by the competitors. The three most critical questions involved the recall, precision, and false positive rate related to assessment of the target behavior. The competitors were given aggregated statistical summary data from a real, but unidentified organization to form the basis of the forecast. The following 5 of the 21 questions for this problem illustrate the variety of questions that the competitors needed to answer.

1. What proportion of users whose data transfer habits differ from their peer group will be alerted by the system, i.e., recall?

2. Of the users who are listed in the alert group, what proportion have data transfer habits that differ from their peer group, i.e., precision?

3. What proportion of users who do not have data transfer habits that differ from their peer group will be in the alert group, i.e., false positive rate?

4. What proportion of users who have web data transfers that differ from their peer group will be in the alert group, i.e., recall?

5. What proportion of users who are in the top $5 \%$ of their peer group in standardized number of sent emails larger than $5 \mathrm{MB}$ will be in the alert group, i.e., recall?

The performance forecasts were then evaluated by comparing them to ground truth (or a detailed expert estimate of ground truth by members of the organization) to assess three measures of accuracy: (a) the mean squared error (MSE) of the prediction, (b) the certainty interval calibration (CIC) assessed as a percentage of correct answers within the $60 \%$ certainty interval, and (c) an interval scoring rule (ISR) which gave a substantial penalty for predictions that were substantially outside of the certainty interval. Lower values of MSE and ISR are better, while the optimal value for CIC is $60 \%$.

The fact that the sponsor had access to data from a single organization limited the number of problems directly related to insider threat that could be developed. Consequently, half of the challenge problems were concerned with target behaviors associated with insider threat variables, while the other half considered more general human resources problems. The eight problems that addressed insider threat focused on variables such as abnormal work habits, large data transfers, unusual foreign contacts, unusual data transfer habits, and changes in personal web use. These problems had the advantage of relevance, but it was more difficult to establish ground truth. The remaining eight problems addressed issues such as employee turnover [Griffeth 2000], movement of employees between work groups, predicting job categories, and line manager identification. Although these problems are less relevant, the value of the target variables could be easily and unambiguously established.

Our approach to solving these challenge problems contained the following four basic steps [Buede 2018]:

1. Data analysis and preparation, with expert elicitation of missing data, as needed. We reviewed the data provided with the problem to determine whether it was sufficient to allow us to generate a population on which we could apply the down-select methods. Where key information was missing, we developed a way to obtain this information from subject-matter experts, using the procedures described in the next section.

2. Population synthesis. We used several methods to simulate the distribution of variables in the challenge problem. As problems got more complicated and involved more variables, some of the methods were not feasible. The primary method for simulating the population was based on the Gaussian copula, using the procedure described in the next section. In addition, we used a discrete event simulation for most problems. We also tried other methods, including stochastic optimization, Markov chain Monte Carlo, neural networks, Bayesian networks, and logistic regression, but these methods were abandoned after one or two problems, either because they didn't scale to more complex problems, or because they didn't produce 
accurate answers to the questions in the problem for which they were used. Three organizations in the project team developed independent population simulations.

3. Down-select algorithm application, per question. The down-select algorithm is the method that the IE uses to identify risk. These methods were provided as a part of the problem statement. We received problems using a variety of down-select methods, including simple summation of component alerts, principal component analysis, DBSCAN clustering, classification trees, neural networks, support vector machines, random forest algorithms, naïve Bayesian networks, and hidden Markov models. We applied the provided methods to the simulated populations and recorded the requested values for recall, precision, and false positive rate.

4. Model fusion with calculation of the mean and $60 \%$ certainty interval, by question. We used a linear opinion pool as a function of the variable being assessed in each question to establish our point and interval estimates. We sometimes adjusted our estimates manually to take some of the features of the scoring rules into account.

Each quarter we received between one and three challenge problems. At the end of the quarter, we submitted our answers, and the accuracy of our forecast (and the forecasts of the other competitors) was determined, using the three criterion measures described previously, MSE, CIC, and ISR. Ideal performance would produce a value of 0.0 for MSE and ISR, and the value of 0.6 for CIC. For MSE, our team had a value of 0.04 , while our competitors had values of 0.20 and 0.12 , respectively. For ISR, our team had a value of 0.44 , while our competitors had values of 1.46 and 0.95 , respectively. Finally, for CIC, our team had a value of 0.64 , while our competitors had values of 0.24 and 0.50 , respectively. For all three criteria, our team had substantially better scores. Consequently, the other two competitors were eliminated from the competition.

Following the completion of the competition, the organization authorized the release of anonymized individual data for one of the challenge problems, and we received these data from the sponsor. This problem involved an IE that determined whether an employee was a line manager, based on their online activity. Although this problem does not involve insider threat, it is a rich problem that included 142 observables measured over 10 months. We have used a subset of these data to conduct the evaluation described in this paper.

\section{POPULATION SIMULATION AND EXPERT ASSESSMENT PROCEDURES}

We first present our approach to simulating proxy data from the joint distribution of target behaviors and their observable indicators from a set of provided statistical properties. Then, for cases in which some statistical properties were not provided, we describe our approach to the elicitation of missing conditional probabilities and correlations from experts.

\subsection{Simulating Populations from Redacted Data}

Our overall project goal was to determine IE performance metrics for a given population and a given algorithm for determining who exhibited a target behavior (e.g., insiders who presented a threat of malicious behavior). One challenge to meeting this goal was that the data we received were redacted so that we received distribution statistics for indicator variables (such as the number of emails sent to foreign addresses) rather than individual cases. Since we couldn't access the original data, we simulated a population that matched the redacted data in the marginal distribution of variables and their intercorrelations.

Thus, the first challenge was to simulate a population of observable indicators and outcome variables over one or more time periods, based on some or all of the following summary information: (a) means and standard deviations of observable variables, by time period, separately for the group of employees exhibiting the target behavior and the group that did not; (b) histograms of observable indicator values by target behavior group, (c) correlations of the values of indicators at a given time, and (d) correlations between values of the same indicator at different times.

Digital Threats: Research and Practice, Vol. 3, No. 1, Article 4. Publication date: October 2021. 
To meet this challenge, we specified a joint distribution of indicators, separately for the group exhibiting the target behavior and the group not exhibiting the target behavior, that matched the statistical properties of the summary information for each group. Sampling from a joint distribution (reflecting the distribution of each indicator value, the target behavior, and the correlations between pairs of indicators) allowed us to construct multiple user populations to account for uncertainty due to the redacted nature of the data.

Simulating correlated non-normal variables can be difficult, but methods have been developed in several different fields to meet the challenge. One of the most popular methods was developed by Vale and Maurelli [1983]. This method starts from a multivariate normal population then applies a polynomial transformation to each random variable based on the first four moments of the distribution [Fleishman 1978]. Because making this transformation changes the correlation of the simulated variables from the target correlation, they solve for an "intermediate" correlation that when specified in the starting multivariate normal distribution will recover the target correlation. Other researchers, most notably Hendrick [2002], have developed similar methods to overcome some of the limitations of the Vail and Maurelli method. In this method the Fleishman polynomial is replaced by a fifth-order polynomial. A recent comparison of the two methods [Olvera Astivia 2015] confirmed some of the benefits of Hendrik's method but suggested that advances in computational power and statistical theory have made possible a wide range of additional methods that might provide better matches to the characteristics of some simulation conditions. We describe two of these methods in the following paragraphs.

The first of these methods is based on the copula, which is a multivariate distribution with uniformly distributed marginal components. A well-known theorem by Sklar [1959] states that any multivariate distribution can be expressed as a function of its marginal distributions and a copula, and that the copula is unique when all random variables are continuous. Conversely, given the marginal distributions and a copula, it is possible to define a joint distribution with the marginals. We used this approach [Nelsen 2006] to specify a joint distribution for each group, based on the descriptive statistics, histograms, and correlations that we received.

The copula approach is a flexible means of constructing a joint distribution, allowing modeling of marginal distributions of random variables and their dependencies separately, often with applications to high dimensional simulation problems. In simulation applications, the approach (described below in more detail) can be viewed as a generalization of the inverse cumulative distribution function (CDF) method, in which the inverse CDF is applied to a uniform distribution (i.e., a random number between 0 and 1) to sample from a target distribution. In the copula approach, a d-dimensional pseudo random vector is sampled from a copula, a multivariate distribution with uniform $(0,1)$ marginal components, then component-wise inverse CDF transformations are applied using the marginal CDFs of the target d-dimensional joint distribution. In this generalization, marginal CDFs are modeled separately and the dependency of the components of the copula distribution are specified such that the component-wise inverse CDF produces the dependency in the target joint distribution.

In our application, we used the Gaussian copula approach to construct the target joint distribution. Because indicators are generally not multivariate normally distributed (in fact, they are often highly skewed), this approach only provides an approximation of their correlations or dependencies. However, because they are separately modeled, we could construct the marginal distributions of indicators with as much accuracy as possible.

In a second method, Ruscio and Kaczetow [2008] developed a procedure that is very similar to a copula approach in that it samples directly from non-normal data by transforming a correlated multivariate normal distribution with a correlation matrix specified to reproduce a target correlation. However, Ruscio and Kaczetow add an iterative procedure to define an "intermediate" correlation matrix to better match the target correlation matrix. We did not incorporate such a method in our approach, but we have used a similar method in subsequent research.

The key steps employed in estimating the joint distribution using the Gaussian copula are described in the following subsections. A more compete mathematical description of the process is shown in the appendix.

3.1.1 Modeling the Marginal Distributions of Indicators. The goal in this step is to construct the inverse $\mathrm{CDFs}$ or quantile functions needed to transform the marginal uniform pseudo random values from the copula

Digital Threats: Research and Practice, Vol. 3, No. 1, Article 4. Publication date: October 2021. 
distribution to random indicator observations. We estimated the marginal distributions by smoothing the histograms of each indicator provided as part of each challenge problem. Depending on whether the histograms had equal bin widths or equal bin proportions, we made the estimates using kernel density estimation or empirical cumulative distribution methods, respectively.

Thus, there are two cases for modeling the marginal distributions of indicators. In the first case, when we received histograms with equal bin widths, we used kernel density estimation to estimate the marginal distribution of each indicator. We used the Gaussian kernel, which allowed the CDF to be estimated as a mixture of normal distributions. In the second case, when we received histograms with equal bin proportions, we used the empirical distribution function, after creating additional "pseudo data" within each bin.

In both cases, we used linear interpolation for greater accuracy by creating equally spread "pseudo data" within each bin, except for the first or last bin, which received special attention. For this bin, we used a beta distribution to distribute the pseudo data points within the bin with parameters $\alpha$ and $\beta$ chosen so that the final estimated marginal distribution would reproduce the provided mean and standard deviation of the indicator with reasonable accuracy. Given the highly skewed nature of many indicators, these are important adjustments to either end of the distribution where target behavior identification is likely sensitive. Finally, we used a smoothing spline to construct continuous quantile functions.

The resulting inverse CDF for each indicator mapped the unform marginal distribution of the copula to the empirical distribution of the indicator.

3.1.2 Modeling the Dependency of Indicators. As previously mentioned, we used the Gaussian copula to model the dependency among indicators. The goal in this step is to construct the copula distribution from which to jointly sample correlated uniform pseudo random numbers. We considered three cases, depending on the number time periods for which measurements were taken and the number of indicators.

In the first case, problems with indicators measured at a single time point, we simply set the indicator correlations in the target joint distribution using the correlations provided in the data, then used packages or custom programs in R (in the copula library) or python to numerically construct the Gaussian copula. Alternatively, we could generate Gaussian copula uniforms by sampling from the multivariate standard normal distribution with the provided detector correlations and transforming the realized random normal values to random uniform values using the normal CDF.

In the second case, problems included indicators measured at multiple time points, but the numbers of indicators and time points were relatively small. The copula includes both correlation among indicators at each time and correlations across time periods. In this case the full correlation matrix is of dimension PxT, where P is the number of indicators and $\mathrm{T}$ is number of time periods. In this case, we used a simple "brute force" approach, modeling the dependency across indicators and time periods in one step using the full PxT correlation matrix. In general, pairwise correlations between different indicators at different times were not available. In this case, we approximated the missing correlations using the correlations between two different indicators at a given time and the auto correlations of each indicator across time points. We estimated the correlation between two different variables at two different times as the product of (a) the arithmetic mean of the correlations between the two variables at each of the two times, and (b) the geometric mean of the autocorrelations of each indicator across the two time periods. When the autocorrelations have opposite signs, then the geometric mean is taken of the absolute value of the autocorrelations, and the sign of the result is reversed. The resulting full correlation matrix is then transformed to the nearest positive-definite matrix. We then used the transformed correlation matrix to set the correlation in the target joint distribution across indicators and time points.

In the third case, there are even larger numbers of time periods $(\mathrm{T})$ and of indicators $(\mathrm{P})$, so that the one-step brute force approach is computationally infeasible. For such problems, we used a more complex approach that sequentially modeled the dependency among indicators one period at a time, conditional on a few earlier time periods (lags). This approach employed standard results involving conditional multivariate normal distributions 
[Anderson 1984] to chain the T lower dimensional Gaussian copulas, with the complete chain of T Gaussian copulas defining the full dependency across indicators and time periods. A detailed description of this method is included in the appendix.

3.1.3 Simulating from the Joint Distribution. The goal of this final step is to construct multiple user populations by simulating them from the estimated joint distribution of indicators and correlations. From the preceding steps we obtained: (a) the quantile functions $Q_{j t}(u)$, each of which transforms a uniform $(0,1)$ random variable to the distribution of the $j^{\text {th }}$ indicator at time $t$; (b) the Gaussian copula $C\left(u_{1,1}, \ldots, u_{P, T}\right)$, one set each for the group exhibiting the target behavior and the group not exhibiting the target behavior. To simulate the total of $N$ users from the joint distribution for a group, we independently sampled $N$ random vectors $U_{i}=\left(u_{1,1, i} \ldots, u_{P, T, i}\right)$ from the Gaussian copula $C, i=1, \ldots, N$, then used the quantile functions to obtain indicator values for the $i^{\text {th }}$ user, $X_{i}=\left(x_{1,1, I}, \ldots, x_{P, T, i}\right)$, where $x_{j, t, I}=Q_{j t}\left(u_{j t}\right)$. The vector-valued observations $X_{1}, \ldots, X_{N}$ comprised a user population and could then be used to answer the forecast questions.

\subsection{Incorporating Expert Judgment}

Some of the problems we received did not provide sufficient information for us to estimate the performance of the IE described in the problems. Two types of information were missing in these problems: (a) information that distinguished the indicator values for users exhibiting the target behaviors from the values for users who did not exhibit that behavior, and (b) pairwise correlations between indicators. Both types of information are required for accurate predictions of IE performance. Since the ability of an indicator to predict the target behavior depends on the difference between the likelihood of the indicator when the target behavior occurs and the likelihood when the behavior is absent, an accurate forecast of IE performance is not possible if the information is missing. Similarly, ignoring indicator intercorrelations in simulating the population can produce unrealistic combinations of indicator values. Furthermore, high indicator intercorrelations can reduce the strength of the inference that can be made regarding the target behavior based on a pattern of indicator values, since their contributions are not independent.

Since information about the behavior of users at a specific organization is not available from published sources, we developed procedures to estimate missing information from the considered judgments about employee online behavior of computer security experts on the project staff. We designed elicitation tools and methods to obtain the required information, employing several procedures to minimize sources of bias, as the following subsections illustrate.

3.2.1 Target Behavior State Conditional on Indicator Value Distributions. To obtain information about the relationship between the distribution of indicators (e.g., after-hours activity) and the target behavior state (e.g., large downloads after hours), we asked experts to assess the likely target behavior state as a function of the values of detected indicator behaviors. This task is analogous to the task that an analyst might be called upon to do in an operational setting; namely to review information about a person and to assess whether that person is a likely threat. In addition, we asked the experts to assess the likelihood that each person (as represented by a simulated profile of indicator values) would show the target behavior.

Both of these judgments are difficult and subject to bias. Assessments of conditional probabilities have been shown to be affected by how the assessments are framed [Gigerenzer 2002]. Specifically, Gigerenzer [2003] noted that conditional probabilities are particularly confusing judgments to make, but presenting these probabilities as frequencies (i.e., numbers of people rather than proportions or probabilities) results in more accurate and coherent judgments. In their review of methods to reduce bias in probability assessments, Chang [2016] confirmed the superiority of frequency formats over probability formats. In addition, displaying probabilities as "nested sets" or visualizing data distributions can improve the accuracy of assessments [Mellers 1999]. We incorporated the

Digital Threats: Research and Practice, Vol. 3, No. 1, Article 4. Publication date: October 2021. 


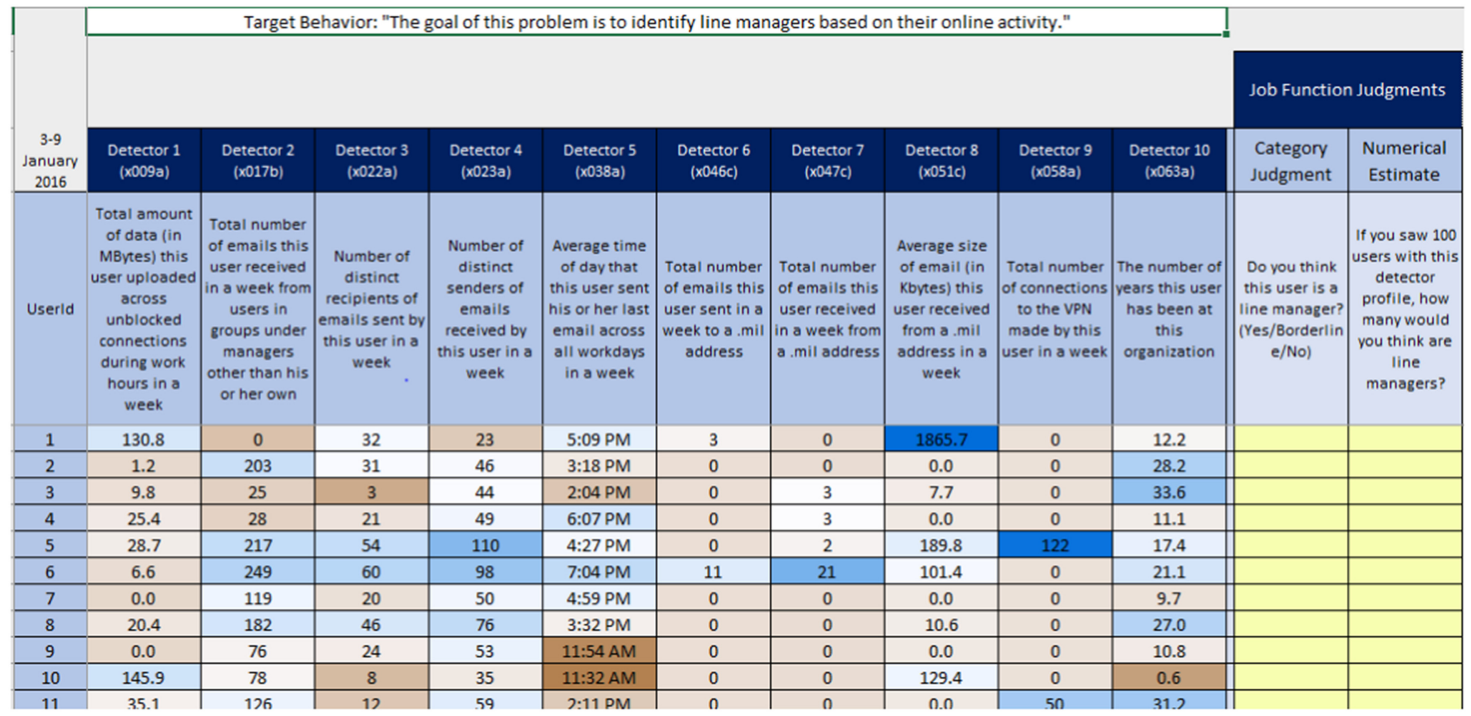

Fig. 1. Excerpt of category identification spreadsheet.

first of these approaches, asking the experts to estimate the number of individuals out of a group of 100 with a similar pattern of indicators who would be expected to exhibit the target behavior.

In addition, we employed elements of process-based options described by Chang [2016] that described common reasoning patterns that lead to biased estimates and explained how to avoid them. In our elicitation procedure, we instructed the experts to look at the marginal distributions of the indicator values, so that they would pay sufficient attention to the base rate (e.g., the number of employees in the population who come in after hours). We provided a complete distribution of indicator values, so that the experts could determine where a given profile of indicators fell relative to the user population. In addition, we provided instructions to highlight that the indicator values provided to the experts were incomplete and uncertain. Finally, the experts were reminded that some individuals might exhibit the target behavior without showing clues in the values of any of the indicators.

We designed a category spreadsheet to present information to experts and to collect their responses. Figure 1 shows a screen image of the spreadsheet that was used in this study. Each row of the spreadsheet represented a single case, consisting of a value for each of several indicators (denoted as a "detector" in the figure, consistent with tournament nomenclature). Experts responded to as many as 150 cases, depending on the complexity of the problem. The cases were selected to be consistent with the provided distributions and intercorrelations between indicator values. We did not have information about how many employees in the population exhibited the target behavior, but we were given information about how many were alerted by the IE. In developing our sample of cases, we oversampled from the segment of the population for which the IE issued alerts. The experts were given access to the distribution of indicator values and could use their own analytic software to visualize the indicator value distributions and to determine how extreme a particular profile was.

For each case, the experts made two estimates. First, they estimated whether or not the profile represented someone who exhibited the target behavior using a three-point scale (yes, borderline, no). Second, they estimated the number of individuals, within a group of 100 with the same pattern of values, who would exhibit the target behavior, following the recommendations in Gigerenzer [2003]. Experts made their estimates individually and then discussed any discrepancies to reach a consensus judgment. Indicator value distributions could then be established for each target behavior group, based on the characteristics of the cases judged to be in each group. 


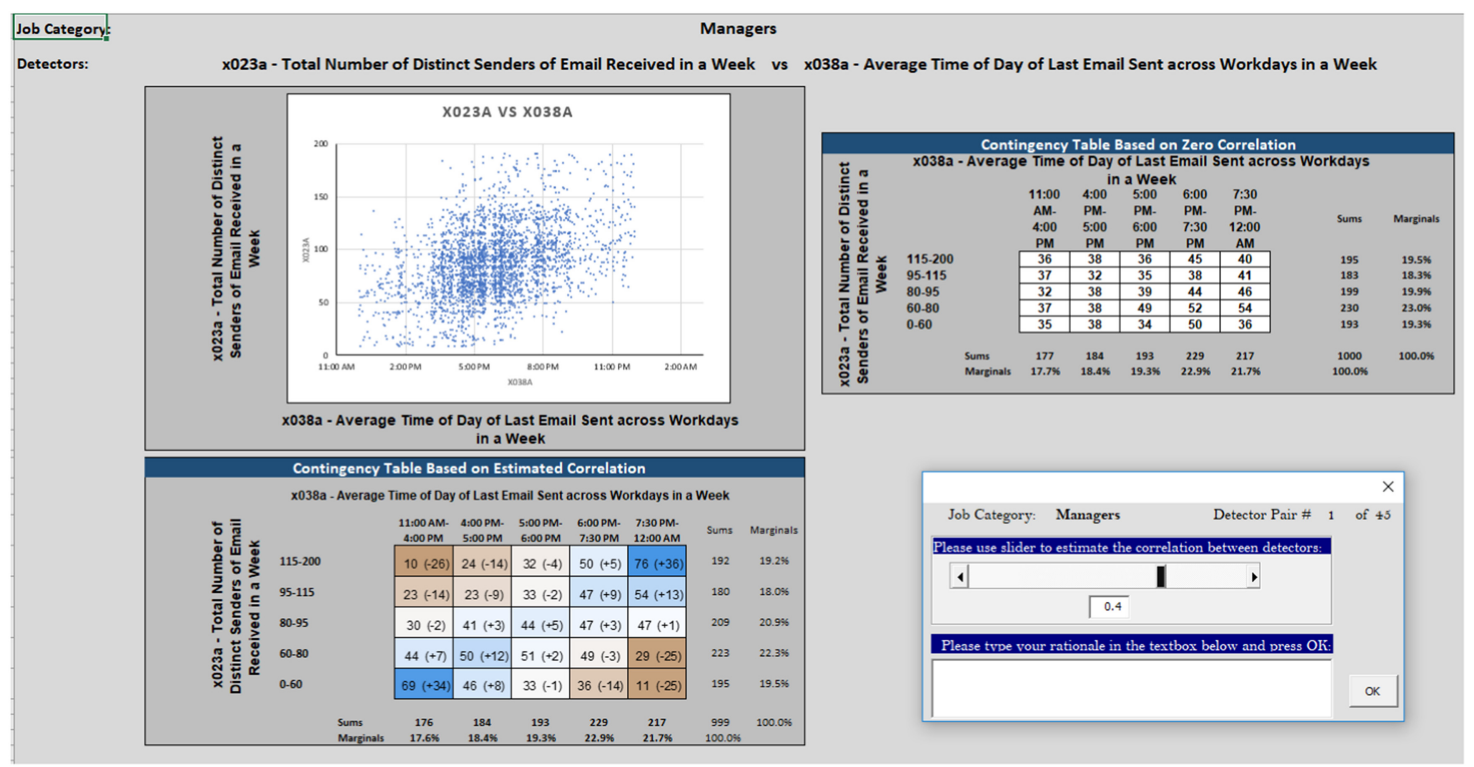

Fig. 2. Graphical and tabular relationship between two indicators with correlation of 0.4 .

3.2.2 Indicator Correlations. In one of the problems we received, indicator intercorrelations were not provided, and we were required to assess them based on expert judgments. It is difficult to estimate correlations, and nonzero correlations are often assessed, even when they are empirically absent [Chapman 1967]. Other research has explained this phenomenon using the availability heuristic, which is the tendency to judge an event as probable to the extent that instances of the event are readily available for recall [Tversky 1973]. According to this explanation, the ability to remember cases in which two variables were both high or both low might lead an analyst to estimate a positive correlation between these two variables, even if none exists.

To minimize the effects of availability, we provided the experts with graphical and tabular representations of the potential correlations between the indicators. Figure 2 shows a screen image depicting the relationship between two indicators, measuring two aspects of email activity. The expert was able to explore as many possible correlation values as desired by moving a slider in the lower right part of the figure that increased and decreased the correlation. For each correlation value selected, the graphical and tabular displays of the joint distribution would be updated while preserving the marginal distributions for each variable. When the expert was satisfied with the joint distribution, he or she would click on a button to record the choice and enter any rationale for the level of correlation selected. The Gaussian copula was used to simulate a joint distribution that matched the marginal distributions of the indicators (as provided in the tournament) and the redacted correlation being estimated by the expert. The graph on the top part of Figure 2 shows a scatterplot of the joint distribution. The table on the bottom is a contingency table that highlights the difference between the distribution and a baseline distribution in which the two indicators are independent. The difference between the two distributions is shown by the numbers in parentheses in the table cells. In addition, the cells are shaded in proportion to the magnitude of the difference. Positive differences are shaded with a blue color, while negative differences are shaded with a brown color. In the example shown in Figure 2 below, the weekly number of people sending emails to an employee and the average time of the last workday email sent to an employee are assessed as having a pairwise correlation of 0.4 , resulting in 25 fewer cases of nighttime emails (7:30 pm-12:00 am) for an employee who receives emails from 60 or fewer recipients than would be expected if there was no association between these indicators.

Digital Threats: Research and Practice, Vol. 3, No. 1, Article 4. Publication date: October 2021. 
The number of correlations to estimate might be large. For example, in a problem with 16 indicators, there are 120 pairwise correlations between them (i.e., 16*15/2). In addition, these correlations need to be estimated for each target behavior state. Estimating this large number of correlations might be beyond the capacity (or beyond the patience) of the experts. In these cases, it might be necessary to sample the pairs of variables for which the correlation will be estimated. Alternatively, one could assume that the correlations don't vary by target behavior state.

The experts would initially estimate each correlation and provide rationale for their estimates. Then, the experts would conduct a meeting to reconcile the differences between them. In that meeting, they would focus primarily on the correlations with large variation in estimates. After reviewing the recorded rationale for each rating, the raters would discuss the rationale to ensure that all had a common understanding and common assumptions regarding the meaning of the variables and how the values of one might affect the other. The discussion would lead raters to change their ratings, which would generally reduce the range of estimates. We estimated the correlations as the median of the post-discussion judgments.

Next, we present a demonstration of our approach to imputing redacted information by comparing the results of our approach to a particular tournament challenge problem with ground truth data that was unavailable during the tournament.

\section{EVALUATION METHOD}

\subsection{Data Source}

In one challenge problem from the tournament, we were tasked with distinguishing who was and who wasn't a manager, based on detected online behavior grouped into indicators. After we responded to the questions in this challenge problem, we were given additional data that would allow us to evaluate the quality of our simulation of the population. We used several experts to assess whether or not simulated cases represented managers, and to estimate the intercorrelations among indicators for both managers and non-managers. Finally, we compared our imputed statistical properties to those of the ground truth dataset provided to us by our sponsor after the tournament.

We used a subset of data from a specific problem from the tournament to evaluate the accuracy (i.e., precision, recall, and false alarm rate) of methods to identify line managers (that is, group managers and assistant managers), based on their on-line activities (see [Buede 2018] for a description of the problems). The data were taken from a real, but unknown, organization and represented a population of 3,304 individuals, including 177 managers and 3,127 non-managers. The data included 142 observable indicators, that described various online activities during a week (such as number of emails sent or received, number of VPN connections, web downloads). The indicators were assessed for 63 weeks between 29 November 2015 and 11 February 2017. We received the following information: (a) the mean and standard deviation for each week for each indicator, separately for managers and non-managers; (b) histograms for each week for each indicator, separately for managers and non-managers; (c) Pearson correlations between indicators for each week, separately for managers and non-managers; and (d) autocorrelations between scores on each indicator for all pairs of weeks, separately for managers and nonmanagers. The subset of the data for this analysis included ten indicators for a single week and was selected using the following procedure.

\subsection{Selecting the Indicators}

We identified potentially important observables using a combined quantitative and qualitative approach. First, we simulated ten preliminary populations using provided organization statistics, using a Gaussian copula approach [Yousefi 2018]. With each population, we trained a random forest classifier and estimated the importance of each observable indicator using the frequency with which the classifier used each observable to make a classification decision. For example, if a random forest classifier used one observable more frequently than another in its 


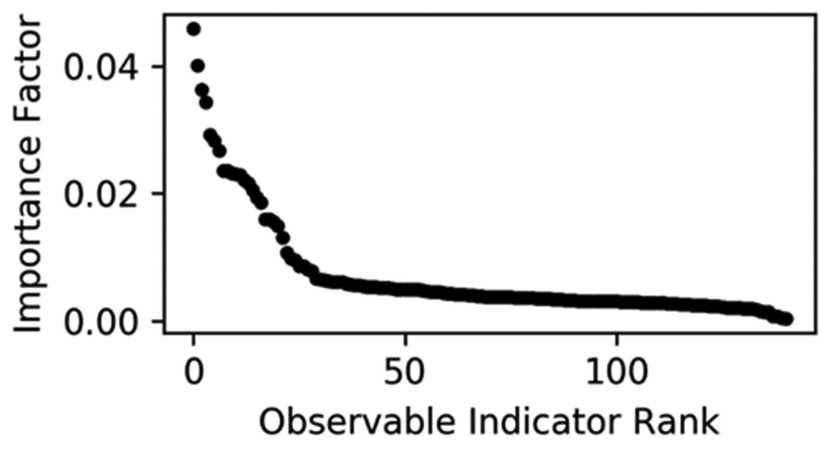

Fig. 3. Distribution of importance of observable indicators.

Table 1. Selected Observables in Order of Decreasing Importance

\begin{tabular}{clc}
\hline Observable ID & \multicolumn{1}{c}{ Observable Name } & $\begin{array}{c}\text { Average } \\
\text { Importance }\end{array}$ \\
\hline$\times 051 \mathrm{c}$ & Average size of email received from external address suffix .mil & 0.0458 \\
$\times 038 \mathrm{a}$ & Average time of day of last email sent across all workdays & 0.0401 \\
$\times 058 \mathrm{a}$ & Total number of connections to the VPN & 0.0363 \\
$\times 047 \mathrm{c}$ & Total number of emails received from external address suffix .mil & 0.0343 \\
$\times 017 \mathrm{~b}$ & Total number of emails received from other work groups & 0.0283 \\
$\times 063 \mathrm{a}$ & Years at organization & 0.0231 \\
$\times 023 \mathrm{a}$ & Number of distinct senders received from all others & 0.0186 \\
$\times 046 \mathrm{c}$ & Total number of emails sent to an external address suffix .mil & 0.0149 \\
$\times 022 \mathrm{a}$ & Number of distinct recipients sent to all others & 0.0096 \\
$\times 009 \mathrm{a}$ & Total amount of data uploaded across unblocked connections & 0.0049 \\
\hline
\end{tabular}

decision nodes, the more frequently occurring observable was considered to be more important than the other. We used the random forest classifier implemented in Sci-Kit Learn version 0.19 .2 with default settings [Pedregosa 2011]. We then averaged each observable's estimated importance across the ten preliminary populations, and ranked the observables using these averages. Figure 3 shows the distribution of average importance. A majority of observables have consistently low importance. A risk with this approach to characterizing feature importance is overlooking the importance of the interaction of features within the random forest classifier trees since we primary look at the cardinality of a feature across the set of decision trees as an indicator of importance. However, we use the feature importance estimations in conjunction with a qualitative process, as described in the next paragraph, so that we are not solely focused on the most "important" features, which can help mitigate the drawbacks of our feature importance estimation approach.

In order to incorporate indicators at different importance levels, we divided the range of importance into ten segments of the same width and selected one indicator nearest the top of each segment. We then reviewed the list of indicators to ensure that they were easy to understand without detailed technical knowledge, and that they covered a range of indicator types (e.g., emails received, emails sent, VPN connections, data transfer, demographics). Based on this review, we made one adjustment to the list to replace an observable from an overrepresented category (i.e., emails received) with an observable with nearly the same importance from an underrepresented category (i.e., data transfer). The ten selected observables are shown, along with their average importance, in Table 1.

Digital Threats: Research and Practice, Vol. 3, No. 1, Article 4. Publication date: October 2021. 
Table 2. Sampling Probabilities by Class and Discriminant Analysis Results

\begin{tabular}{llcccccccc}
\hline & & \multicolumn{7}{c}{ Probability of Manager Class from Discriminant Analysis } & \multirow{2}{*}{ Total } \\
\cline { 3 - 9 } & & $\mathbf{0 . 0 - 0 . 1}$ & $\mathbf{0 . 1 - 0 . 2}$ & $\mathbf{0 . 2 - 0 . 4}$ & $\mathbf{0 . 4 - 0 . 6}$ & $\mathbf{0 . 6 - 0 . 8}$ & $\mathbf{0 . 8 - 0 . 9}$ & $\mathbf{0 . 9}-\mathbf{1 . 0}$ & \\
\hline \multirow{3}{*}{ Non- } & Count in Population & 310 & 1416 & 741 & 314 & 182 & 108 & 56 & 3127 \\
managers & \% within Class & $9.9 \%$ & $45.3 \%$ & $23.7 \%$ & $10.0 \%$ & $5.8 \%$ & $3.5 \%$ & $1.8 \%$ & $100.0 \%$ \\
& Sample Size & 4 & 48 & 27 & 10 & 6 & 3 & 2 & 100 \\
& Sampling Percentage & $4.0 \%$ & $48.0 \%$ & $27.0 \%$ & $10.0 \%$ & $6.0 \%$ & $3.0 \%$ & $2.0 \%$ & $100.0 \%$ \\
\hline \multirow{3}{*}{ Managers } & Count in Population & 2 & 7 & 17 & 27 & 44 & 49 & 31 & 177 \\
& \% within Class & $1.1 \%$ & $4.0 \%$ & $9.6 \%$ & $15.3 \%$ & $24.9 \%$ & $27.7 \%$ & $17.5 \%$ & $100.0 \%$ \\
& Sample Size & 1 & 2 & 5 & 7 & 14 & 16 & 5 & 50 \\
& Sampling Percentage & $2.0 \%$ & $4.0 \%$ & $10.0 \%$ & $14.0 \%$ & $28.0 \%$ & $32.0 \%$ & $10.0 \%$ & $100.0 \%$ \\
\hline
\end{tabular}

\subsection{Selecting the Time Period}

We selected a single week to provide data for the experts to rate. We wanted to use a week that was early in the range of weeks for which we had data that was representative of the total time period. Because the data started at the end of November 2015, the first several weeks included the Christmas and New Year's Day holidays, which we believed were not typical weeks for online behavior. Consequently, we chose 3-9 January 2016 as the target week for our analyses. An informal review of the means of the indicators by week confirmed our conjecture that the earlier weeks were atypical.

\subsection{Simulating the Population}

Based on the indicator histograms, descriptive statistics, and indicator correlations for the selected week, we used the Gaussian copula to generate a sample of 5,000 managers and 5,000 non-managers, overrepresenting managers in our synthetic sample. We then conducted a linear discriminant analysis using known group membership (manager or not) to construct a set of predictive linear combinations of indicator variables in order to calculate a score for probability of being a manager for any case. We saved the model output showing the probability of being a manager for each case to use to define strata for sampling. We then randomly selected 177 cases from the simulated manager sample, and 3,127 cases from the simulated non-manager sample, to match the population of the organization. The predicted probability of being a manger was 0.28 for the simulated non-managers, and 0.69 for the simulated managers.

We then selected a sample of 150 cases to present to the experts. We partitioned the cases according to their class (manager vs. non-manager) and the probability of being a manager from the discriminant analysis. The probability was partitioned into seven categories with the following cutpoints: $0.1,0.2,0.4,0.6,0.8$, and 0.9 . We over-sampled managers, to produce a sample of 50 manager cases and 100 non-manager cases. We also undersampled some of the more obvious cases, that is, managers with a model probability of being a manager of greater than 0.9 and non-managers with a model probability of less than 0.1 . The sampling probabilities are shown in Table 2.

We reviewed three indicators related to sent emails to ensure that they had consistent values for cases that sent no emails. Specifically, there were nine cases in which indicator $\times 022 \mathrm{a}$, the number of distinct recipients of emails sent by this user in a week, had the value of zero, indicating that no emails were sent. For these cases, we set $\times 046$, the total number of emails this user sent in a week to a .mil address, to zero. In addition, we set $\times 038$ a, the average time of day that this user sent his or her last email across all workdays in a week, to "NA." When we analyzed the data, we restored the value of $\times 038$ a to the missing data value of zero (signifying midnight), to correspond to the way that variable was coded in the original dataset that we were given. It was not possible to recode the original data to distinguish missing data from true zeros. 
The cases in the sample were provided to the participants, who assessed whether or not the cases represented managers. In a second exercise, the participants estimate the correlations among the indicators, separately for managers and non-managers, based on the marginal distributions of the indicators in the population.

\subsection{Participants}

In order to elicit correlations between online behaviors for managers and non-managers from subject matter experts, we recruited six participants with expertise and experience in business administration, industrialorganizational psychology, and information science from two of our author's organizations. We selected recently hired or promoted program managers, as well as an individual who recently transitioned from management to a principal scientist role, so that they would have recent experience of the differences in online requirements of management and non-management tasks. Three were Industrial-Organizational psychologists; one had a business background, and two had information technology backgrounds.

\subsection{Procedure}

We began by getting informed consent forms and some demographic information from our participants. Then there were four steps in the procedure: We asked the participants to (a) review the set of 150 cases and estimate whether the case represented a manager or non-manager; (b) meet as a group to discuss ratings and resolve differences; (c) estimate correlations between indicators separately for managers and non-managers; and (d) meet as a group to discuss correlation estimates and resolve differences.

4.6.1 Estimation of Manager/Non-manager. Each participant received a category identification spreadsheet to record his or her estimate (See Figure 1). Each row of the spreadsheet represented a single case, consisting of a value for each of the 10 indicators. There was a total of 150 cases. Participants answered the following two questions for each case. First, they were asked if they thought the user represented in the case was a line manager. Participants were allowed the following three responses: yes, borderline, and no. A borderline response indicated that the participant thought that the data did not demonstrate unequivocally whether or not the person in the case was a line manager. Second, they were asked that if they saw 100 users with this indicator profile, how many they would think were line managers [Gigerenzer 2003].

The participants were reminded that these indicators only present partial information about the user in each case and were encouraged to consider all of the reasons that either managers or non-managers might exhibit the pattern of indicator values presented in each case. The participants were encouraged to use the spreadsheet to make calculations to aid their judgment. Two of the participants added a worksheet that they used to conduct their own calculations to support their decisions.

The spreadsheet included other information to help the participants understand the case data. The mean and standard deviation of each indicator in the simulated population was provided, and the indicator values in the display were color coded according to their value, compared to the mean. A blue background indicated that the value was above the mean, while a tan background indicated that it was below the mean. In addition, in a separate spreadsheet, the standard scores were presented numerically for each indicator value in each case. Finally, the spreadsheet provided the percentiles of the distribution for each indicator.

4.6.2 Meeting of Participants to Reconcile Differences. To accommodate the busy schedules of the participants, we limited the meeting to reconcile discrepancies across rater judgments to two hours. To ensure that we got as much useful information as possible, we prioritized the cases with the most disagreement. To determine the level of disagreement, we recoded the category judgments on a scale where -1 represented a judgment that the case was a non-manager, 0 represented a judgment of borderline, and 1 represented a judgment that the case was a manager. We then calculated the standard deviation of the recoded judgments for each case. We ordered the cases by standard deviation, from the maximum to the minimum and discussed cases in this order.

Digital Threats: Research and Practice, Vol. 3, No. 1, Article 4. Publication date: October 2021. 
We prepared a summary spreadsheet that aggregated the individual category judgments and numerical estimates of the participants. This spreadsheet was projected on a screen so that all participants' ratings could be seen and discussed. At the beginning of the session, each participant gave a brief description of the method he or she used to make estimates. Then the participants discussed the cases in order. The participants stated the rationale for their judgments, and the group would discuss the cases. Usually, after the discussion, one or more of the participants would change their category judgments, so that the post-discussion category judgments were often unanimous. However, when there was still some disagreement, the group settled differences by majority choice. Toward the end of the meeting, we reviewed the remaining cases to determine whether there were any that could not easily be resolved by majority choice. One such case was discovered, which the participants then discussed to resolve their disagreement.

4.6.3 Indicator Correlation Estimation. Using the elicitation tool shown in Figure 2, each participant individually estimated the correlation between each pair of indicators, separately for managers and non-managers, for a total of 90 correlation estimates. In addition, the participants were encouraged to provide text indicating their rationale for their estimates. The extent of the rationale provided varied greatly from almost none to a sentence or two for each estimate. Participants estimated the correlation for each pair of indicators twice, once for nonmanagers and once for managers. Each time a participant completed five indicator pairs (for 10 estimates) he or she was given an opportunity to take a break, until all 45 pairs were completed.

4.6.4 Meeting of Participants to Reconcile Differences in Correlation Estimates. When the participants had returned their completed spreadsheets, we combined their estimates in a single spreadsheet. As in the case above, because of the busy work schedules of the participants, the meeting was restricted to approximately two hours. Consequently, we focused the discussion on the indicator pairs for which there was the most disagreement.

We sorted the pairs of variables by several measures of the magnitude of the difference in the estimates between participants. We calculated the standard deviation and the range of the correlation estimates across raters, separately for managers and non-managers. We then calculated an index of disagreement that was based on the maximum of these four measures. We ranked the indicator pairs by this index and color coded all the disagreement measures to make it easy for the facilitator to identify the source of disagreement in the estimates,

The participants were given a copy of their rationale to help them in the discussion. Discussion typically consisted of participants describing why they made the estimate that they made. The facilitator asked those with minority opinions to provide their rationales to ensure that all points of view were expressed. After considering the rationale of other participants, the participants were given the opportunity to change their estimates. The facilitator recorded any revised estimates on an aggregation spreadsheet.

When the meeting was nearly over, the facilitator reviewed the remaining un-discussed pairs and determined that the differences were small enough, so that these items didn't need to be discussed.

\section{EVALUATION RESULTS}

\subsection{Evaluation of the Estimates Produced by the Gaussian Copula Method}

We compared the indicator variable means and standard deviations in the population simulated using the Gaussian copula to the comparable statistics for the actual population provided by our sponsor after the tournament. Both the simulated and actual populations contained 177 managers and 3,127 non-managers. The simulated cases were drawn from a larger simulated sample of 5,000 managers and 5,000 non-managers. Because the distributions of indicator variables were highly skewed, we used a bootstrapping approach to test the statistical significance of differences between the populations. The bootstrap method was applied separately for non-managers and managers. For each group, we used $\mathrm{R}=1,000$ replications (each replication resampled with replacement from the larger sample). We constructed the bootstrap distribution of differences $\left(\hat{\theta}_{r}^{*}-\hat{\theta}\right), r=1, \ldots, R$ where $\hat{\theta}_{r}^{*}$ is the mean or SD computed from the $r$ th resample and $\hat{\theta}$ is the mean or SD computed from the larger sample 
Table 3. Comparison of Simulated and Actual Indicator Means and Standard Deviations

\begin{tabular}{|c|c|c|c|c|c|c|c|c|c|c|}
\hline \multirow[b]{2}{*}{ Var. } & \multicolumn{2}{|c|}{ Simulated Data } & \multicolumn{2}{|c|}{ Actual Data } & \multicolumn{3}{|c|}{ Mean Differences } & \multicolumn{3}{|c|}{ Std. Dev. Differences } \\
\hline & Mean & Std. Dev. & Mean & Std. Dev. & Difference & Effect Size & p value & Difference & Diff. Ratio & $\mathrm{p}$ value \\
\hline \multicolumn{11}{|c|}{ Class $=$ Non-Managers } \\
\hline $009 \mathrm{a}$ & $51,582,645$ & $122,824,314$ & $44,622,748$ & $124,716,898$ & $6,959,897$ & 0.057 & 0.003 & $-1,892,584$ & -0.015 & 0.795 \\
\hline k017b & 86.37 & 139.86 & 78.87 & 137.27 & 7.49 & 0.054 & 0.002 & 2.60 & 0.019 & 0.743 \\
\hline$\times 022 \mathrm{a}$ & 27.10 & 28.11 & 25.71 & 27.15 & 1.39 & 0.049 & 0.005 & 0.96 & 0.034 & 0.216 \\
\hline$\times 023 a$ & 40.28 & 34.03 & 39.14 & 32.36 & 1.14 & 0.034 & 0.049 & 1.67 & 0.049 & 0.015 \\
\hline$\times 038 \mathrm{a}$ & 0.58 & 0.20 & 0.58 & 0.20 & -0.01 & -0.033 & 0.074 & 0.00 & 0.009 & 0.614 \\
\hline \begin{tabular}{l|l}
$\times 046 c$ \\
\end{tabular} & 3.60 & 11.83 & 3.09 & 10.93 & 0.50 & 0.043 & 0.008 & 0.90 & 0.076 & 0.191 \\
\hline$\times 047 \mathrm{c}$ & 2.42 & 7.83 & 2.09 & 8.36 & 0.33 & 0.043 & 0.017 & -0.53 & -0.068 & 0.390 \\
\hline \begin{tabular}{l|}
$\times 051 \mathrm{c}$ \\
\end{tabular} & 61,667 & 309,230 & 51,511 & 301,270 & 10,155 & 0.033 & 0.063 & 7,961 & 0.026 & 0.752 \\
\hline \begin{tabular}{r|}
$\times 058 \mathrm{a}$ \\
\end{tabular} & 6.99 & 25.94 & 6.65 & 26.29 & 0.34 & 0.013 & 0.471 & -0.35 & -0.014 & 0.835 \\
\hline \begin{tabular}{r|}
$\times 063 a$ \\
\end{tabular} & 13.01 & 12.37 & 13.19 & 12.33 & -0.18 & -0.015 & 0.412 & 0.04 & 0.004 & 0.792 \\
\hline \multicolumn{11}{|c|}{ Class = Managers } \\
\hline$\times 009 a$ & $56,438,123$ & $237,107,749$ & $45,156,917$ & $200,382,978$ & $11,281,206$ & 0.048 & 0.520 & $36,724,771$ & 0.155 & 0.605 \\
\hline$\times 017 \mathrm{~b}$ & 158.54 & 73.35 & 157.63 & 76.66 & 0.90 & 0.012 & 0.879 & -3.31 & -0.045 & 0.387 \\
\hline$\times 022 \mathrm{a}$ & 59.56 & 23.61 & 56.62 & 26.71 & 2.94 & 0.124 & 0.101 & -3.10 & -0.131 & 0.019 \\
\hline$\times 023 \mathrm{a}$ & 85.99 & 32.91 & 86.83 & 33.17 & -0.84 & -0.026 & 0.752 & -0.27 & -0.008 & 0.879 \\
\hline$\times 038 \mathrm{a}$ & 0.73 & 0.16 & 0.72 & 0.14 & 0.00 & 0.024 & 0.731 & 0.02 & 0.122 & 0.403 \\
\hline$\times 046 \mathrm{c}$ & 18.22 & 25.80 & 18.53 & 27.32 & -0.31 & -0.012 & 0.881 & -1.52 & -0.059 & 0.574 \\
\hline \begin{tabular}{l|}
$\times 047 \mathrm{c}$ \\
\end{tabular} & 12.07 & 14.63 & 11.09 & 14.44 & 0.98 & 0.067 & 0.401 & 0.18 & 0.013 & 0.887 \\
\hline \begin{tabular}{l|}
$051 \mathrm{c}$ \\
\end{tabular} & 124,204 & 253,794 & 125,762 & 335,456 & $-1,558$ & -0.006 & 0.933 & $-81,662$ & -0.322 & 0.199 \\
\hline \begin{tabular}{r|}
$058 \mathrm{a}$ \\
\end{tabular} & 39.79 & 57.60 & 35.69 & 56.41 & 4.10 & 0.071 & 0.333 & 1.20 & 0.021 & 0.839 \\
\hline$\times 063 a$ & 16.20 & 8.69 & 16.24 & 8.23 & -0.04 & -0.005 & 0.954 & 0.46 & 0.053 & 0.429 \\
\hline
\end{tabular}

$(\mathrm{n}=5,000)$ which was obtained using our population generation method based on the Gaussian copula. These difference replications were compared against $\left(\hat{\theta}-\theta_{0}\right)$, where $\theta_{0}$ is the mean or SD from the actual data. The idea here is that the bootstrap distribution of differences $\left(\hat{\theta}_{r}^{*}-\hat{\theta}\right)$ resembles the sampling distribution of $\left(\hat{\theta}-\theta_{0}\right)$ under the null hypothesis $H_{0}: \theta=\theta_{0}$ [Hall 1991]. In our analysis the p-value is the proportion of replications that produced $\left|\hat{\theta}_{r}^{*}-\hat{\theta}\right|>\left|\hat{\theta}-\theta_{0}\right|$. With the large number of cases in the non-manager group, relatively small differences might be significant. Consequently, we also calculated the effect size for mean differences and the difference ratio (i.e., the ratio of the difference and the actual value) for standard deviation differences.

The means and standard deviations of the simulated and actual data are shown in Table 3. As was anticipated, there were significant differences $(\mathrm{p}<0.05)$ between six of the ten variable means for the non-manager population, while there were no statistically significant differences between variable means for the manager population. However, the effect sizes related to the significant mean differences were quite small, with absolute values less than 0.057 . Thus, the differences, though they are statistically significant, are practically inconsequential.

The standard deviations of the indicators in the simulated data were also very close to the comparable values in the actual population. Significant differences $(p<0.05)$ were found in just 2 of the 20 values. The probability of outcome at least this extreme if there is no difference between the simulated and actual data is 0.26 . The difference ratio shows the difference between the standard deviations of the two populations as a proportion of the simulated standard deviation. The small magnitude of these differences indicate that the simulation procedures closely match the actual distributions.

We applied a similar bootstrap procedure to assess how well the simulated and actual indicator correlations matched. The results of this analysis, shown in Table 4, indicate that indicator correlations differ substantially 
Table 4. Comparison of Simulated and Actual Indicator Correlations

\begin{tabular}{|c|c|c|c|c|c|c|c|c|c|c|c|}
\hline \multirow[b]{2}{*}{$\begin{array}{l}\text { Row } \\
\text { Var. }\end{array}$} & \multirow[b]{2}{*}{$\begin{array}{c}\text { Column } \\
\text { Var. }\end{array}$} & \multicolumn{5}{|c|}{ Non-Managers } & \multicolumn{5}{|c|}{ Managers } \\
\hline & & $\begin{array}{l}\text { Sim. } \\
\text { Corrs. }\end{array}$ & $\begin{array}{l}\text { Act. } \\
\text { Corrs. }\end{array}$ & $\begin{array}{l}\text { Corr. } \\
\text { Diff. }\end{array}$ & $\begin{array}{c}\text { Corr } \\
\text { Diff. } \\
\text { Ratio }\end{array}$ & value & $\begin{array}{l}\text { Sim. } \\
\text { Corrs }\end{array}$ & $\begin{array}{l}\text { Act. } \\
\text { Corrs }\end{array}$ & $\begin{array}{l}\text { Corr. } \\
\text { Diff. }\end{array}$ & $\begin{array}{l}\text { Corr. } \\
\text { Diff. } \\
\text { Ratio }\end{array}$ & p value \\
\hline$\times 017 \mathrm{~b}$ & $\times 009 a$ & -0.0019 & 0.0274 & -0.0293 & 1.0698 & 0.011 & -0.1335 & -0.0462 & -0.0873 & 1.8905 & 0.174 \\
\hline$\times 022 a$ & $\times 009 a$ & -0.0076 & 0.0353 & -0.0430 & 1.2159 & 0.013 & -0.0896 & 0.0171 & -0.1068 & 6.2371 & 0.117 \\
\hline$\times 023 a$ & $\times 009 a$ & 0.0197 & 0.0319 & -0.0122 & 0.3814 & 0.428 & -0.1272 & -0.0246 & -0.1025 & 4.1646 & 0.135 \\
\hline$\times 038 \mathrm{a}$ & $\times 009 a$ & 0.0684 & 0.0662 & 0.0022 & 0.0333 & 0.879 & -0.0685 & 0.0416 & -0.1101 & 2.6459 & 0.055 \\
\hline$\times 046 c$ & $\times 009 a$ & 0.0063 & 0.0300 & -0.0237 & 0.7898 & 0.162 & -0.0489 & 0.0721 & -0.1210 & 1.6787 & 0.014 \\
\hline$\times 047 \mathrm{c}$ & $\times 009 a$ & -0.0213 & 0.0073 & -0.0286 & 3.9389 & 0.013 & -0.0451 & 0.0148 & -0.0599 & 4.0547 & 0.143 \\
\hline$\times 051 \mathrm{c}$ & $\times 009 a$ & 0.0151 & 0.0557 & -0.0405 & 0.7283 & 0.031 & 0.0547 & 0.1607 & -0.1061 & 0.6599 & 0.138 \\
\hline$\times 058 \mathrm{a}$ & $\times 009 a$ & -0.0071 & -0.0019 & -0.0052 & 2.7700 & 0.666 & -0.0525 & 0.1002 & -0.1527 & 1.5239 & 0.042 \\
\hline$\times 063 a$ & $\times 009 a$ & -0.0039 & -0.0349 & 0.0309 & 0.8870 & 0.063 & 0.0576 & -0.0282 & 0.0858 & 3.0404 & 0.124 \\
\hline$\times 022 a$ & $\times 017 \mathrm{~b}$ & 0.2870 & 0.3739 & -0.0869 & 0.2325 & 0.001 & 0.5280 & 0.6593 & -0.1313 & 0.1991 & 0.011 \\
\hline$\times 023 a$ & $\times 017 b$ & 0.4094 & 0.5377 & -0.1284 & 0.2387 & 0.000 & 0.7629 & 0.8709 & -0.1081 & 0.1241 & 0.003 \\
\hline$\times 038 \mathrm{a}$ & $\times 017 b$ & 0.1247 & 0.2245 & -0.0998 & 0.4445 & 00 & 0.2960 & 0.4321 & -0.1362 & 0.3151 & .013 \\
\hline$\times 046 c$ & $\times 017 \mathrm{~b}$ & 0.1071 & 0.1114 & -0.0043 & 0.0389 & 0.861 & 0.2503 & 0.3378 & -0.0875 & 0.2590 & 0.235 \\
\hline$\times 047 \mathrm{c}$ & $\times 017 b$ & 0.0731 & 0.1134 & -0.0403 & 0.3554 & 0.184 & 0.2882 & 0.3834 & -0.0951 & 0.2482 & 0.142 \\
\hline$\times 051 \mathrm{c}$ & $\times 017 b$ & -0.0150 & 0.0064 & -0.0214 & 3.3297 & 0.052 & -0.0371 & 0.0097 & -0.0468 & 4.8332 & 0.452 \\
\hline & & & & & & & & & & & 309 \\
\hline$\times 063 a$ & $\times 017 b$ & 0.0696 & 0.1030 & -0.0334 & 0.3245 & 0.054 & 0.2046 & 0.1056 & 0.0990 & 0.9377 & 0.108 \\
\hline$\times 023 a$ & $\times 022 a$ & 0.6375 & 0.7370 & -0.0995 & 0.1350 & 0.000 & 0.6513 & 0.7219 & -0.0706 & 0.0979 & 0.160 \\
\hline$\times 038 \mathrm{a}$ & & & 0. & & & & & 97 & & & 03 \\
\hline$\times 046 c$ & $\times 022 a$ & 0.1813 & 0.3452 & -0.1639 & 0.4749 & 0.000 & 0.4283 & 0.5360 & -0.1077 & 0.2009 & 0.074 \\
\hline$\times 047 \mathrm{c}$ & $\times 022 a$ & 0.0791 & 0.1966 & -0.1175 & 0.5975 & 0.000 & 0.2419 & 0.4196 & -0.1777 & 0.4236 & 0.012 \\
\hline$\times 051 \mathrm{c}$ & $\times 022 a$ & -0.0150 & 0.0204 & -0.0354 & 1.7377 & 5 & -0.0732 & 0.0571 & -0.1 & 2.2813 & 023 \\
\hline$\times 058 \mathrm{a}$ & $\times 022 a$ & 0.0404 & 0.1188 & -0.0784 & 0.6601 & & 0.1980 & 0.2092 & -0.0112 & 0.0537 & 894 \\
\hline$\times 063 a$ & $\times 022 \mathrm{a}$ & 0.0201 & 0.0210 & -0.0009 & 0.0429 & 0.963 & 0.0081 & -0.0773 & 0.0854 & 1.1048 & 0.305 \\
\hline$\times 038 \mathrm{a}$ & $\times 023 a$ & 0.2988 & 0.4476 & -0.1488 & 0.3324 & 0.000 & 0.3373 & 0.4385 & -0.1012 & 0.2308 & 0.149 \\
\hline$\times 046 c$ & $\times 023 a$ & 0.1652 & 0.2339 & -0.0 & 0.2939 & & 0.3053 & 0.3376 & -0.0 & 0.0959 & 0.669 \\
\hline$\times 047 \mathrm{c}$ & $\times 023 a$ & 0.0843 & 0.1793 & -0.0950 & 0.5299 & & 0.2240 & 0.2668 & -0.0428 & 0.1605 & 0.545 \\
\hline$\times 051 c$ & $\times 023 a$ & 0.0091 & 0.0365 & -0.0274 & 0.7508 & 0.171 & -0.0333 & 0.0410 & -0.0743 & 1.8129 & 0.185 \\
\hline$\times 058 \mathrm{a}$ & $\times 023 a$ & 0.1111 & 0.1658 & -0.0546 & 0.3296 & 0.026 & 0.2650 & 0.3133 & -0.0483 & 0.1541 & .453 \\
\hline$\times 063 a$ & $\times 023 a$ & 0.1780 & 0.2370 & -0.0590 & 0.2488 & 0.001 & 0.1580 & 0.1306 & 0.0274 & 0.2099 & 0.692 \\
\hline$\times 046 c$ & $\times 038 \mathrm{a}$ & 0.0802 & 0.1767 & -0.0965 & 0.5461 & 0.000 & 0.1318 & 0.2208 & -0.0890 & 0.4030 & 0.031 \\
\hline$\times 047 \mathrm{c}$ & $\times 038 \mathrm{a}$ & 0.0306 & 0.1296 & -0.0990 & 0.7639 & 0.000 & 0.0878 & 0.1884 & -0.1006 & 0.5338 & 0.041 \\
\hline$\times 051 \mathrm{c}$ & $\times 038 a$ & 0.0169 & 0.0505 & -0.0336 & 0.6656 & 0.083 & 0.0506 & 0.0309 & 0.0197 & 0.6359 & 0.548 \\
\hline$\times 058 \mathrm{a}$ & $\times 038 a$ & 0.0323 & 0.1331 & -0.1009 & 0.7577 & 0.000 & 0.1477 & 0.1594 & -0.0117 & 0.0734 & 0.759 \\
\hline$\times 063 a$ & $\times 038 a$ & -0.0222 & -0.0679 & 0.0457 & 0.6728 & 0.005 & -0.1010 & -0.1523 & 0.0512 & 0.3365 & 0.585 \\
\hline$\times 047 \mathrm{c}$ & $\times 046 c$ & 0.2901 & 0.5820 & -0.2919 & 0.5015 & 0.000 & 0.6480 & 0.7520 & -0.1040 & 0.1383 & 0.043 \\
\hline$\times 051 \mathrm{c}$ & $\times 046 c$ & 0.0507 & 0.1371 & -0.0864 & 0.6300 & 0.000 & 0.0141 & 0.1224 & -0.1083 & 0.8845 & 0.117 \\
\hline$\times 058 \mathrm{a}$ & $\times 046 c$ & 0.0511 & 0.1508 & -0.0997 & 0.6614 & 0.000 & 0.0881 & 0.1171 & -0.0291 & 0.2481 & 0.658 \\
\hline$\times 063 a$ & $\times 046 c$ & -0.0100 & 0.0397 & -0.0497 & 1.2507 & 0.013 & 0.1040 & -0.0408 & 0.1448 & 3.5479 & 0.137 \\
\hline$\times 051 \mathrm{c}$ & $\times 047 \mathrm{c}$ & 0.0340 & 0.1130 & -0.0789 & 0.6987 & 0.000 & 0.0506 & 0.1334 & -0.0829 & 0.6209 & 0.230 \\
\hline
\end{tabular}

(Continued) 
Table 4. Continued

\begin{tabular}{|c|c|c|c|c|c|c|c|c|c|c|c|}
\hline \multirow[b]{2}{*}{$\begin{array}{l}\text { Row } \\
\text { Var. }\end{array}$} & \multirow[b]{2}{*}{$\begin{array}{c}\text { Column } \\
\text { Var. }\end{array}$} & \multicolumn{5}{|c|}{ Non-Managers } & \multicolumn{5}{|c|}{ Managers } \\
\hline & & $\begin{array}{l}\text { Sim. } \\
\text { Corrs. }\end{array}$ & $\begin{array}{l}\text { Act. } \\
\text { Corrs. }\end{array}$ & $\begin{array}{l}\text { Corr. } \\
\text { Diff. }\end{array}$ & $\begin{array}{c}\text { Corr } \\
\text { Diff. } \\
\text { Ratio }\end{array}$ & p value & $\begin{array}{l}\text { Sim. } \\
\text { Corrs }\end{array}$ & $\begin{array}{l}\text { Act. } \\
\text { Corrs }\end{array}$ & $\begin{array}{l}\text { Corr. } \\
\text { Diff. }\end{array}$ & $\begin{array}{c}\text { Corr. } \\
\text { Diff. } \\
\text { Ratio }\end{array}$ & p value \\
\hline$\times 058 \mathrm{a}$ & $\times 047 \mathrm{c}$ & 0.0582 & 0.1115 & -0.0533 & 0.4780 & 0.053 & 0.2154 & 0.1539 & 0.0615 & 0.3995 & 0.440 \\
\hline$\times 063 \mathrm{a}$ & $\times 047 \mathrm{c}$ & 0.0069 & 0.0515 & -0.0446 & 0.8659 & 0.005 & 0.0519 & -0.1013 & 0.1532 & 1.5123 & 0.050 \\
\hline$\times 058 \mathrm{a}$ & $\times 051 \mathrm{c}$ & 0.0026 & 0.0321 & -0.0295 & 0.9205 & 0.055 & 0.0039 & 0.1447 & -0.1408 & 0.9732 & 0.061 \\
\hline$\times 063 a$ & $\times 051 \mathrm{c}$ & 0.0073 & 0.0196 & -0.0124 & 0.6299 & 0.464 & -0.0422 & -0.0586 & 0.0163 & 0.2789 & 0.730 \\
\hline$\times 063 \mathrm{a}$ & $\times 058 \mathrm{a}$ & 0.0408 & 0.0737 & -0.0330 & 0.4470 & 0.081 & -0.0318 & 0.0077 & -0.0395 & 5.1546 & 0.521 \\
\hline
\end{tabular}

Table 5. Accuracy Metrics for Expert

Category Assessments

\begin{tabular}{lc|cc}
\hline \multicolumn{2}{c|}{ Initial Estimates } & \multicolumn{2}{c}{ Consensus Estimate } \\
\hline Rater & Cramer's V & Measure & Value \\
\hline Rater 1 & $0.668^{* *}$ & Cramer's V & $0.603^{* *}$ \\
Rater 2 & $0.417^{* *}$ & Recall & 0.700 \\
Rater 3 & $0.579^{* *}$ & Precision & 0.761 \\
Rater 4 & $0.396^{* *}$ & False Positive & 0.110 \\
Rater 5 & $0.682^{* *}$ & & \\
Rater 6 & $0.330^{* *}$ & & \\
Average & 0.512 & & \\
Median & 0.498 & & \\
\hline
\end{tabular}

${ }^{* *} \mathrm{p}<0.01$

between the simulated and actual data. The simulated and actual correlations were significantly different for 28 of the 45 indicator pairs among non-managers, and for 11 pairs among managers. On average, simulated correlations were about 0.06 lower than actual correlations for non-managers, and about 0.05 lower for managers. This difference ranges from -0.29 to 0.15 . The correlation difference ratio, which is the correlation difference divided by the actual correlation, show that there are 22 indicator pairs among non-managers, and 32 pairs among managers, in which the simulated correlation is more than twice or less than half of the actual value. However, this ratio should be interpreted with some caution, because low actual correlations will produce a high ratio even when the actual difference is relatively small. Despite these differences, there is a good general agreement between the simulated and actual correlations, as indicated by correlations between these actual and estimated values of 0.96 for non-managers, and 0.94 for managers.

\subsection{Accuracy of Expert Category Estimates}

Since the category judgments had three levels (no, borderline, yes), while the actual data had two levels (manager, non-manager), we assessed the accuracy of the expert judgments using Cramer's V. As the results in the left panel of Table 5 show, each of the experts was able to identify managers from the observable indicators with reasonable accuracy. Correlations between individual estimates and the actual group represented by each case, assessed using Cramer's V, were all significantly greater than zero. The group consensus performed better than the average or median of the individual assessments, but not as well as the best two individual estimates. Measures of recall and precision, and the false positive rate, were also all relatively high, as shown on the right panel of Table 5. 
Table 6. Indicator Means for Actual Groups and Consensus Judgments

\begin{tabular}{|c|c|c|c|c|c|c|c|}
\hline & \multicolumn{3}{|c|}{ Actual Groups } & \multicolumn{3}{|c|}{ Consensus Judgment } & \multirow[b]{2}{*}{$\begin{array}{c}\text { Group } \\
\text { Comparison }\end{array}$} \\
\hline Variable & $\begin{array}{c}\text { Non- } \\
\text { Managers }\end{array}$ & Managers & Ratio & $\begin{array}{c}\text { Non- } \\
\text { Managers }\end{array}$ & Managers & Ratio & \\
\hline$\times 009 a$ & 32.53 & 96.57 & 2.97 & 36.30 & 33.90 & 0.93 & 3.18 \\
\hline$\times 017 \mathrm{~b}$ & 90.18 & 158.51 & 1.76 & 72.44 & 227.76 & 3.14 & 0.56 \\
\hline$\times 022 \mathrm{a}$ & 20.44 & 57.66 & 2.82 & 18.73 & 45.63 & 2.44 & 1.16 \\
\hline$\times 023 a$ & 39.18 & 83.41 & 2.13 & 33.39 & 92.59 & 2.77 & 0.77 \\
\hline$\times 038 \mathrm{a}$ & 0.68 & 0.76 & 1.11 & 0.68 & 0.74 & 1.08 & 1.03 \\
\hline$\times 046 c$ & 3.24 & 18.60 & 5.74 & 3.36 & 8.46 & 2.51 & 2.28 \\
\hline$\times 047 a$ & 2.24 & 10.51 & 4.70 & 2.21 & 5.63 & 2.55 & 1.84 \\
\hline$\times 051 \mathrm{a}$ & 63.84 & 117.84 & 1.85 & 69.45 & 49.71 & 0.72 & 2.58 \\
\hline$\times 058 \mathrm{a}$ & 8.93 & 58.15 & 6.51 & 7.01 & 40.05 & 5.71 & 1.14 \\
\hline$\times 063 a$ & 14.82 & 15.02 & 1.01 & 13.81 & 21.24 & 1.54 & 0.66 \\
\hline
\end{tabular}

Table 6 shows the means of indicator variables by group for both the actual groups and the consensus judgments. If the differences between the actual manager and non-manager means are similar to the corresponding differences in the simulated manager and non-manager means, then the consensus judgments could provide reasonable predictions of group membership, compared to the actual groups. Although there are no standard guidelines for interpreting this table, we took a group comparison value of greater than 2.0 or less than 0.5 as indicating a potential problem in the correspondence between the simulated and actual groups. The group comparison values were outside of this range for two variables, $\times 009$ a (Total amount of data uploaded across connections) and $\times 046 \mathrm{c}$ (Total number of emails sent to an external address suffix .mil). For $\times 009 \mathrm{a}$, the actual managers had substantially higher values than actual non-managers, but those judged by the experts to be managers had roughly the same value as those judged to be non-managers. A similar pattern, although not as extreme, pattern was found for $\times 046 \mathrm{c}$. These results indicate that expert judgments can provide a reasonable surrogate for actual data when those data are not available.

\subsection{Accuracy of Expert Correlation Estimates}

Table 7 provides a summary of the performance of the experts in estimating observable indictor correlations. The first column of this table shows the pre-discussion correlation between the estimated and actual indictor correlation coefficients. These correlations show that there is a wide range in performance for this estimation task. The best two experts (Raters 2 and 3) gave estimates that correlated over 0.80 with the actual values for nonmanagers, and over 0.66 for managers. On the other hand, the worst two experts (Raters 4 and 6 ) had estimates that had negative correlations with actual values for non-managers and were 0.12 or less for managers. A similar picture is painted by the pre-discussion mean squared error (MSE) values, which vary from 0.01 to 0.42 for non-manager estimates and from 0.02 to 0.26 for the manager estimates.

The median of the pre-discussion estimates provides a very good estimate of the correlations, nearly as good as the best two raters for non-manager estimates, and between the best and second-best raters for the manager estimates. The average pre-discussion rating also provides a relatively good estimate, but except for the postdiscussion estimates for managers, the median performed noticeably better than the average.

Comparison of the pre-discussion and post-discussion values in Table 7 show a modest improvement in performance, at best, following the discussion between the raters. The average correlation improves with discussion, but the median changes little and is actually a little bit lower after discussion than before. Table 8 presents an ANOVA of the MSE values to quantify the effects of discussion, group, and case (i.e., indicator pair) on error. 
Table 7. Accuracy and Error Measures of Correlation Estimates

\begin{tabular}{|c|c|c|c|c|c|c|c|c|}
\hline \multirow[b]{3}{*}{ Estimate } & \multicolumn{4}{|c|}{ Pre-Discussion } & \multicolumn{4}{|c|}{ Post-Discussion } \\
\hline & \multicolumn{8}{|c|}{ Non-Managers } \\
\hline & Correlation & MSE total & $\begin{array}{c}\text { MSE } \\
\text { Discussed }\end{array}$ & $\begin{array}{c}\text { MSE not } \\
\text { Discussed }\end{array}$ & Correlation & $\begin{array}{l}\text { MSE } \\
\text { total }\end{array}$ & $\begin{array}{c}\text { MSE } \\
\text { Discussed }\end{array}$ & $\begin{array}{c}\text { MSE not } \\
\text { Discussed }\end{array}$ \\
\hline Rater 1 & 0.196 & 0.097 & 0.093 & 0.106 & 0.219 & 0.061 & 0.041 & 0.106 \\
\hline Rater 2 & $0.802^{* *}$ & 0.014 & 0.010 & 0.022 & $0.756^{* *}$ & 0.015 & 0.012 & 0.022 \\
\hline Rater 3 & $0.800^{* *}$ & 0.016 & 0.017 & 0.014 & $0.815^{* *}$ & 0.015 & 0.015 & 0.014 \\
\hline Rater 4 & -0.095 & 0.231 & 0.278 & 0.125 & -0.135 & 0.108 & 0.100 & 0.125 \\
\hline Rater 5 & $0.396^{* *}$ & 0.085 & 0.099 & 0.054 & $0.505^{* *}$ & 0.038 & 0.031 & 0.054 \\
\hline Rater 6 & -0.096 & 0.415 & 0.430 & 0.382 & 0.169 & 0.185 & 0.095 & 0.382 \\
\hline Average & $0.485^{* *}$ & 0.025 & 0.019 & 0.039 & $0.540^{* *}$ & 0.024 & 0.017 & 0.039 \\
\hline Median & $0.744^{* *}$ & 0.014 & 0.008 & 0.028 & $0.704^{* *}$ & 0.017 & 0.012 & 0.028 \\
\hline \multicolumn{9}{|c|}{ Managers } \\
\hline Rater 1 & 0.126 & 0.077 & 0.082 & 0.064 & 0.095 & 0.071 & 0.074 & 0.064 \\
\hline Rater 2 & $0.872^{* *}$ & 0.015 & 0.010 & 0.024 & $0.822^{* *}$ & 0.019 & 0.017 & 0.024 \\
\hline Rater 3 & $0.660^{* *}$ & 0.056 & 0.044 & 0.084 & $0.681^{* *}$ & 0.048 & 0.032 & 0.084 \\
\hline Rater 4 & 0.052 & 0.255 & 0.311 & 0.131 & 0.061 & 0.116 & 0.110 & 0.131 \\
\hline Rater 5 & $0.549^{* *}$ & 0.069 & 0.068 & 0.073 & $0.605^{* *}$ & 0.047 & 0.035 & 0.073 \\
\hline Rater 6 & 0.122 & 0.259 & 0.302 & 0.164 & $0.357^{*}$ & 0.093 & 0.060 & 0.164 \\
\hline Average & $0.548^{* *}$ & 0.044 & 0.042 & 0.049 & $0.614^{* *}$ & 0.039 & 0.035 & 0.049 \\
\hline Median & $0.617^{* *}$ & 0.039 & 0.031 & 0.057 & $0.611^{* *}$ & 0.040 & 0.032 & 0.057 \\
\hline
\end{tabular}

${ }^{*} \mathrm{p}<0.05$

$* * \mathrm{p}<0.01$.

Some estimates were not discussed, so that estimates did not change after the discussion. Consequently, this analysis considers only those estimates that were discussed by the raters. The results shown in Table 8 confirm that there was no significant improvement between the pre- and post-discussion squared error measures following the discussion.

\section{DISCUSSION}

Organizations that use data to identify threats and potential opportunities need to assess the quality of their prediction methods. This need is especially salient in issues related to security, such as the identification of insider threats. However, privacy concerns often limit the information that is available to evaluators to make this assessment. Some of these limitations apply not only to external evaluators, but to many employees of the organization, as well. The methods described in this paper provide a way to simulate a population that approximates the marginal distributions of variables and their intercorrelations to assess the performance of the prediction methods. Our review focused on the quality of the simulation; the ability to use this simulation as a part of a process to assess the performance of an IE has been demonstrated elsewhere [Buede 2018] and is a topic of ongoing research efforts. However, the accuracy of the IEMs that we developed in this project, especially when compared to the performance of the other competitors, speaks well to the utility and accuracy of the simulated populations. We realize that there are several reasons for the success of these models, but that success of the modeling effort would not have been possible if we could not apply the models to a relatively accurate representation of the joint distribution of indicator and target variables within the organization.

Our statistical simulation methods provided an accurate estimate of the means and standard deviations of observable indicators. Although there are some significant differences between simulated and actual values of 
Table 8. ANOVA Results for Squared Error of Correlation Estimates

\begin{tabular}{|c|c|c|c|c|c|c|}
\hline Source & & $\begin{array}{c}\text { Type III Sum } \\
\text { of Squares }\end{array}$ & df & $\begin{array}{c}\text { Mean } \\
\text { Square }\end{array}$ & $\mathbf{F}$ & Sig. \\
\hline \multirow[t]{2}{*}{ Intercept } & Hypothesis & 0.099 & 1 & 0.099 & 14.160 & 0.001 \\
\hline & Error & 0.210 & 30 & $.007^{\mathrm{a}}$ & & \\
\hline \multirow[t]{2}{*}{ PrePost } & Hypothesis & 0.001 & 1 & 0.001 & 2.501 & 0.124 \\
\hline & Error & 0.008 & 30 & $.000^{\mathrm{b}}$ & & \\
\hline \multirow[t]{2}{*}{ Manager } & Hypothesis & 0.012 & 1 & 0.012 & 3.645 & 0.066 \\
\hline & Error & 0.103 & 30 & $.003^{\mathrm{c}}$ & & \\
\hline \multirow[t]{2}{*}{ Case } & Hypothesis & 0.210 & 30 & 0.007 & 2.152 & 0.024 \\
\hline & Error & 0.087 & 26.574 & $.003^{\mathrm{d}}$ & & \\
\hline PrePost* $^{*}$ & Hypothesis & 0.000 & 1 & 0.000 & 0.653 & 0.425 \\
\hline Manager & Error & 0.013 & 30 & $.000^{\mathrm{e}}$ & & \\
\hline \multirow[t]{2}{*}{ PrePost $^{*}$ Case } & Hypothesis & 0.008 & 30 & 0.000 & 0.613 & 0.907 \\
\hline & Error & 0.013 & 30 & $.000^{\mathrm{e}}$ & & \\
\hline \multirow[t]{2}{*}{ Manager* Case } & Hypothesis & 0.103 & 30 & 0.003 & 7.952 & 0.000 \\
\hline & Error & 0.013 & 30 & $.000^{\mathrm{e}}$ & & \\
\hline PrePost $^{*}$ & Hypothesis & 0.013 & 30 & 0.000 & & \\
\hline Manager* Case & Error & 0.000 & 0 &.$^{f}$ & & \\
\hline \multicolumn{7}{|c|}{$\begin{array}{l}\text { a. MS(Case). } \\
\text { b. MS(PrePost }{ }^{\star} \text { Case). } \\
\text { c. MS(Manager* Case). } \\
\text { d. MS(PrePost }{ }^{*} \text { Case) }+ \text { MS(Manager }{ }^{*} \text { Case) - MS(PrePost }{ }^{*} \text { Manager }^{*} \text { Case). } \\
\text { e. MS(PrePost* Manager }{ }^{*} \text { Case). } \\
\text { f. MS(Error). }\end{array}$} \\
\hline
\end{tabular}

indicator variables, the magnitude of these differences is small. This result is to be expected from a method based on copulas, because the marginal distributions are estimated directly from the histograms, so the primary source of error is deviation of the distribution from the assumptions of the simulation within histogram categories. Some modest improvement might be obtained, if desired, by increasing the number of categories in the histograms, or by developing more accurate models of the distribution of cases within histogram categories. The methods provide less accurate estimates of indicator variable correlations. This result is also not surprising since the accuracy of the simulated correlations is affected by non-normality of the variables. The error could be reduced by incorporating an "intermediate correlation," such as was used by Ruscio and Kaczetow [2008]. In some of our more recent work, we have used similar methods.

As a case study, the generality of our results is limited. Our analysis considered a single organization and the prediction of a single target variable using a specified analysis method. Although we have some information from our overall performance on the project that the methods can provide relatively accurate results for a variety of target variables and analysis methods, all are data from a single organization. In addition, the simulation represents linear relationships between indicator variables and does not capture non-linear or higher order relationships. This is a theoretical limitation of the method with unknown practical implications, but we suspect that some analysis methods may focus on higher-order interactions that our population simulation methods would not be able to duplicate. In this study, we had to recode some variables in the cases rated by the experts, because they included impossible combinations of indicators values (e.g., no emails sent, but some emails sent to mil addresses). We anticipate it will remain a task for the analyst to identify and address such interactions between indicator variables. 
When information about the distributions of indicator variables for different levels of the target variable (e.g., manager or non-manager, level of insider threat) is not available, expert judgments can provide reasonable surrogate information that can be used to evaluate the performance of an IE. Discussion by the experts increased the accuracy of the elicited estimates, possibly because they shared information that some of them had not considered in making their individual ratings. Our informal observations of the discussions showed that the experts were sometimes very willing to change their ratings when they received new information. This observation would suggest that it is probably better to use the post-discussion judgments as the basis for modeling rather than basing predictions on pre-discussion opinions. However, models could be based on individual, post-discussion ratings, nominal group scores representing a median rating, or the consensus post-discussion rating. We used individual expert models with some success in some of the challenge problems. In addition, the success of our multi-modeling approach [Buede 2018] suggests that there might be a benefit to developing multiple individual models, each representing a single expert. However, our data do not answer the question of whether multiple individual models would be better or worse than a single aggregated model.

The picture regarding expert estimate of correlations between indicators is more complex. There was a wide range of accuracy of the correlation estimates between raters, and performance did not improve significantly with rater discussions. The best raters did very well in estimating correlations, while the worst raters had chance-level performance. One implication of this result is that selection of experts is important to assure accurate estimates. When we reviewed the characteristics of the raters who were the most accurate, we found that they were people with a background in industrial-organization psychology who understood the factors that can affect the value of a correlation, such as restriction of range or unreliability in the measurement of the individual variables. We might also consider other factors in evaluating the experts, such as the characteristics of superforecasters [Tetlock 2015] that have been identified in recent research. In addition to serving as a basis for selection, this information could be used to weight the expert estimates to potentially improve the accuracy of the aggregated forecast. For our expert correlation estimates, the performance of the median of pre-discussion estimates indicates that such a mechanical combination rule might have benefits over post-discussion ratings. Although the quality of group judgments has been a topic for research over many years, there is probably some benefit to be obtained from additional research targeted to the specific judgments involved in assessing the effects of indicator variables and their correlations.

Given the limited ability of some of the experts to estimate correlations, it is reasonable to ask whether there might be other ways for experts to participate that would provide useful and accurate information, and perhaps require less of their time. For example, an expert might be relatively good in estimating whether a correlation was positive, negative, or zero, even though their magnitude estimates were inaccurate. A correlation matrix could then be constructed to match the signs, but to contain nominal magnitude values (which would be adjusted to produce a positive definite correlation matrix). This procedure would reduce precision but would still avoid the problem of overweighting multiple pieces of evidence on highly correlated variables. Further research might identify other ways to use expert judgment more efficiently. In addition, it would be useful to know the effect that use of these simplified correlations would have on the ability to forecast IE performance, which might be relatively insensitive to small changes in the estimated correlations.

The fact that the problem for which we received anonymized individual data involved the identification of managers, rather than being directly related to insider threat necessitates a discussion of the relevance of these results to insider threat. The evaluation of the performance of our IEMs [Buede 2018] showed that both MSE and ISR were substantially lower (i.e., better) for the general human resource problems than for the insider threat problems. Performance on the manager identification problem was not as good as it was for other human resource problems; our models overestimated recall and precision and underestimated the false positive rate. There are differences between these problem classes that might account for the general differences in performance. First, the insider threat problems were given early in the project, while we were developing our methods. Since our performance generally improved as our processes were developed, it is natural that our accuracy would be better

Digital Threats: Research and Practice, Vol. 3, No. 1, Article 4. Publication date: October 2021. 
for the later problems. Second, for many of the insider threat problems, ground truth was determined by experts from the organization, which adds another source of error to the insider threat problems, which would account for some of the performance differences. However, these general differences do not account for the relatively poor performance on the manager identification problem. Thus, although there are differences between the two problem domains in performance, those differences do not suggest that our results do not apply to insider threat problems.

We think that the strongest argument for applying our results to problems dealing with insider threats is that the indicators used in the human resource problems are similar to those used in the insider threat problems. In fact, they come from the same database that was used to generate the problems that were directly related to insider threats. Most of them relate to computer network and online activity, such as remote network logins, copying and printing files, number of emails sent, types of email recipients, and characteristics of internet activity. Others relate to timesheet issues, such as hours worked, variability in schedule, and changes in work schedule. We don't think that there is an inherent difference between sending email to a recipient with a military address (an indicator of being a manager at the subject organization) and sending an email to a recipient with a foreign address (an indicator of espionage) that would imply that different methods are required to simulate the likelihood of these activities.

The fact that the true value of target variables for most of the insider threat problems was determined by experts at the organization, and some of our models for insider threat problems used the judgment of experts on the research team, brings up the possibility that the accuracy of our models might be enhanced by the effects of common expert stereotypes of the importance of insider threat indicators. The fact that both our analysis and the analysis of the experts at the organization rely on a common database that does not reflect human judgment data tends to reduce the likelihood of such an effect, as does the fact that we did not rely on expert judgments for most of our problems. Nevertheless, we cannot rule out the possibility that our performance scores have been inflated by common expert stereotypes. If that were the case, one might expect that we would be better at predicting IE performance for the problems involving expert ratings, compared to problems that did not require expert ratings. However, in general, our models performed better for the human resource problems with more objective criteria, than for the insider threat problems with criteria based on expert judgments [Buede 2018]. Consequently, although we can't rule out the role of expert stereotypes in model accuracy, there isn't evidence for a substantial effect.

Neither our approach to simulating the population described in this paper, nor our approach to model IEs [Buede 2018] contains a psychological model of the process by which insiders are motivated to conduct malicious activities, and the steps in which they plan and prepare to carry them out. In the challenge problems that we solved, the organization did not release any data that could be used to build or calibrate such a model. Despite this fact, we were able to forecast the performance of IEs addressing a variety of target behaviors with relatively high accuracy. Nevertheless, we believe that incorporating such a model could provide two substantial improvements to the utility of our methods. First, incorporating a psychological model can make our approach better able to address issues, such as the motivations and intentions of the employee, which are critical in determining whether a person poses a threat. Since the challenge problems we received in the competition did not address these issues, our approach was able to support accurate assessments of IE performance without a psychological model. However, answering the most critical questions regarding insider threat will require knowledge of psychological constructs. Second, we believe that a psychological model can help suggest new indicator variables that should be included in IEs that are concerned with insider threats. Furthermore, we believe that most organizations collect information on variables that would support the use of such as model.

Our population simulation methods are included as part of a multi-modeling approach to evaluating IE performance. Such an approach is expensive, in that it requires subject-matter experts and multiple modeling teams to implement. It is likely that not all of the features of our methods will be highly effective, and there might be situations in which an organization should conduct their own analysis using internal experts and analysts 
who have complete access to the data, rather than redacting the data for analysis by an outside group. There are several factors of our approach that could be investigated, including the number of modeling teams, the number of forecasts made by each team, the population simulation approaches used, and the method used to aggregate the individual forecasts. This information is a topic for future research and is needed to inform recommendations regarding the best ways to evaluate IE performance. However, the results of our analysis, and the results presented by Buede [2018] indicate that if an organization does not have in-house evaluation capability, and wants to maintain strict confidentiality over their data, they can still get some useful information from an analysis of redacted data.

Predicting insider threats is hard because of its rarity because many of the precursor activities are covert or hard to detect, because there are likely several different paths to malicious insider behavior, and for a host of other reasons that have been enumerated in the research literature. Making accurate inferences requires an understanding of how likely various potential indicators are, both singly and in combination. We believe that the simulation methods that we have described can provide a basis for useful assessments of the performance of inference enterprises that seek to identify potential insider threats.

\section{APPENDIX}

\section{A POPULATION DATA GENERATION METHODS}

This appendix describes methods that were used to construct a population model based on redacted data.

\section{A.1 Copula Approach to Data Simulation}

We simulated population data for each problem using a method based on copulas [Nelsen 2006]; a copula is a multivariate distribution with uniformly distributed marginal components. This method is based on Sklar's Theorem Sklar [1959] which states that the joint distribution of $d$ random variables can be expressed as a function of its marginal distributions $F_{i}, i=1, \ldots, d$ and some copula $C\left(u_{1}, \ldots, u_{d}\right)$ so that

$$
F\left(x_{1}, \ldots, x_{d}\right)=C\left(F_{1}\left(x_{1}\right), \ldots, F_{d}\left(x_{d}\right)\right)
$$

Given the marginal distributions $F_{i}$ 's and a copula $C\left(u_{1}, \ldots, u_{d}\right), F\left(x_{1}, \ldots, x_{d}\right)$ is a joint distribution with marginals $F_{i}$ 's. We used this idea to construct a joint distribution of detectors as summarized below.

Data Simulation Algorithm Using Copula Method

Step 1: Specify a copula $C\left(u_{1}, \ldots, u_{d}\right)$ that approximates detector dependencies.

Step 2: Generate a $d$-variate realization $\left(u_{1}, \ldots, u_{d}\right)$ from $C\left(u_{1}, \ldots, u_{d}\right)$.

Step 3: Transform realized $u_{i}$ s to detector scale using inverse CDFs: $x_{i}=F_{i}^{-1}\left(u_{i}\right)$.

Step 4: Repeat Steps 2 and $3 n$ times indepedently to generate $n$ user/employee observations.

\section{A.2 Detector Marginal Distributions}

This section describes methods employed to estimate detector marginal distributions. The goal of this process is to obtain the inverse CDF or quantile function $F_{i}^{-1}$.

A.2.1 Kernel Density Estimation. The first method used to estimate detector marginal distribution is kernel density estimation (KDE). Given sample data $X_{1}, \ldots, X_{n}$ the kernel density estimator with kernel $K$ and bandwidth $h$ is:

$$
\hat{f}(x)=\frac{1}{n h} \sum_{i=1}^{n} K\left(\frac{x-x_{i}}{h}\right) .
$$

We used the Gaussian kernel $K(x)=\phi(x)$ with bandwith selected to reasonably fit the histogram. Because values of detectors are provided using bins (or class intervals), a number of options are possible for evaluating

Digital Threats: Research and Practice, Vol. 3, No. 1, Article 4. Publication date: October 2021. 
$f(x)$. The simplest option is to evaluate the estimator at the bin midpoints. Using the Gaussian kernel with band width $h$, the estimator is:

$$
\hat{f}(x)=\frac{1}{n h} \sum_{i=1}^{I} n_{i} \phi\left(\frac{x-m_{i}}{h}\right)
$$

where $m_{i}$ 's are the bin midpoints and $n_{i}$ 's are bin sizes, $I$ is number of bins and $n=\sum n_{i}$. Another option is to uniformly spread out the $n_{i}$ osbservations within the $i$ th bin and evaluate the estimator at each pseudo sample data point.

The cumulative distribution function (CDF) corresponding to the estimator with Gaussian kernel is

$$
\begin{aligned}
\hat{F}(x) & =\frac{1}{n h} \int_{-\infty}^{x} \sum \phi\left(\frac{v-m_{i}}{h}\right) d v \\
& =\frac{1}{n} \sum_{i=1}^{l} n_{i} \Phi\left(\frac{x-m_{i}}{h}\right)
\end{aligned}
$$

where $\Phi()$ is the standard normal CDF. Note that this a mixture of normals and that the CDF can be evaluated without actually estimating the kernel density estimate.

We implemented a quantile or inverse CDF function by evaluating $\hat{F}(x)$ over a range of detector values to obtain $\left(x_{i}, \hat{F}\left(x_{i}\right)\right)$ and used spline interpolation to map probability values to detector values.

A.2.2 Empirical Distribution Function. Detector marginal distributions were also estimated using a method that is equivalent to an empirical CDF. This method was preferred over KDE when the provided histograms used bins with varying widths and equal proportions. For this method we created $n_{i}$ equally spaced data points within the $i$ th bin. Non-equal spacing was optionally considered for the last bin, because data points in the last bin are unlikely to be uniform distributed if the sample maximum observation is extremely high. Then sample quantiles were calculated using the pseudo sample data points for a range of probabilities. Using spline interpolation, probability values were mapped to detector values to define the quantile function.

\section{A.3 Detector Dependency: Single or Relatively Low Number of Time Periods}

A.3.1 Scenario 1: Single Time Period. This is the simplest scenario involving few detectors and single time period (no autocorrelation). A straightforward application of Gaussian copula suffices in this scenario as described earlier: define a Gaussian copula $C\left(u_{1}, \ldots, u_{d} ; R\right)$ where $\mathrm{R}$ is the specified detector correlations; generate uniform random values using the copula; transform the random uniform values to detector values (in standardized $\mathrm{Z}$ or original X scale) using detector quantile functions.

A.3.2 Scenario 2: Multiple Time Periods. In this scenario complete detector correlations for each time period are provided, but the correlation between time periods is only provided for the same detector (i.e., autocorrelation). The total dimension also does not present computational difficulty using a straightforward application of Gaussian copula. This scenario also involves approximating the full correlation matrix across detectors and time periods, but otherwise the remainder of the approach is equivalent to that in Scenario 1. In the discussion below, $T$ is number of time periods and $p$ is number of detectors.

Partition the full correlation matrix as

$$
R=\left(\begin{array}{cccc}
R_{11} & R_{12} & \cdots & R_{1 T} \\
R_{21} & R_{22} & \cdots & R_{2 T} \\
\vdots & \vdots & \ddots & \vdots \\
R_{T 1} & R_{T 2} & \cdots & R_{T T}
\end{array}\right)
$$


where $R_{k k}$ is the detector correlation matrix in time period $k$ and $R_{k l}$ is the detector correlation matrix between time periods $k$ and $l$. All correlations in $R_{k k}$ are provided but only the diagonal correlations in the $R_{k l}$ 's are provided.

Denote elements in $R_{k l}$ by $r_{k l}^{i j}$; this is the correlation between detector $i$ in time $k$ and detector $j$ in time $l$. A reasonable approximation for the off-diagonal correlations is

$$
\hat{r}_{k l}^{i j}=\frac{1}{2}\left(r_{k k}^{i j}+r_{l l}^{i j}\right) \sqrt{r_{k l}^{i i} r_{k l}^{j j}}
$$

That is, $r_{k l: i j}$ is the product of (1) the arithmetic mean of the correlation between detectors $i$ and $j$ across time periods $k$ and $l$ and (2) the geometric mean average of the autocorrelations of the two detectors across the two time periods. If $r_{k l}^{i i} r_{k l}^{j j}<0$ the second factor was evaluated as $-\sqrt{\mathrm{abs}\left(r_{k l}^{i i} r_{k l}^{j j}\right)}$.

The approximate detector correlation matrix between time periods $k$ and $l$ can be expressed in terms of provided correlation matrices $R_{k k}$ and diagonal autocorrelation matrix $R_{k l}^{d}=\operatorname{diag}\left(r_{k l}^{11}, r_{k l}^{22}, \ldots, r_{k l}^{p p}\right), k \neq l$ as

$$
\hat{R}_{k l}=\frac{1}{2} \operatorname{sgn}\left(R_{k l}^{d}\right) \operatorname{abs}\left(R_{k l}^{d}\right)^{\frac{1}{2}}\left(R_{k k}+R_{l l}\right) \operatorname{abs}\left(R_{k l}^{d}\right)^{\frac{1}{2}} \operatorname{sgn}\left(R_{k l}^{d}\right)
$$

where the signum and absolute value functions are applied elementwise.

These approximations produce full correlation matrix below representing dependencies across detectors and time periods, which in turn may need additional transformation to be positive definite.

$$
\hat{R}=\left(\begin{array}{cccc}
R_{11} & \hat{R}_{12} & \cdots & \hat{R}_{1 T} \\
\hat{R}_{21} & R_{22} & \cdots & \hat{R}_{2 T} \\
\vdots & \vdots & \ddots & \vdots \\
\hat{R}_{T 1} & \hat{R}_{T 2} & \cdots & R_{T T}
\end{array}\right)
$$

A.3.3 Scenario 3: High Number of Time Periods. In this scenario complete detector correlations for each time period and autocorrelations for the same detector are provided, with a total dimension that makes the "brute force" approach employed in Scenario 2 computationally infeasible.

The overall strategy employed is to chain multiple lower dimensional Gaussian copulas representing dependencies of overlapping subsets of detector-time dimension. This chaining process produces a Gaussian copula that can approximate the full detector-time period dependencies.

Sequence of Conditional Multivariate Normal Distributions. We begin by presenting a sequence of conditional multivariate normal distributions used to construct a Gaussian copula algorithm for simulating detector values in lower dimension one time period at a time. The discussion below employs a compact index notation using $Z_{s: t}, s<t$, to represent $\left(Z_{s}, Z_{s+1}, \ldots, Z_{t-1}, Z_{t}\right)$.

Let $Z_{t}$ be a $p$ dimensional random vector and $Z_{1: T}$ a $T p$ dimensional random vector constructed by concatenating $Z_{1}, \ldots, Z_{T}$. If $Z_{1: T}$ is distributed as multivariate normal with covariance equal to the correlation matrix $R$ in Scenario 2, then conditional on the past $L$ time periods,

$$
Z_{t} \mid Z_{(t-L):(t-1)}=z_{(t-L):(t-1)} \sim N\left(\mu_{t \mid(t-L):(t-1)}, \Sigma_{t \mid(t-L):(t-1)}\right)
$$

where

$$
\begin{aligned}
& \mu_{t \mid(t-L):(t-1)}=R_{t,(t-L):(t-1)} R_{(t-L):(t-1),(t-L):(t-1)}^{-1} z_{(t-L):(t-1)} \\
& \sum_{t \mid(t-L):(t-1)}=R_{t t}-R_{t,(t-L):(t-1)} R_{(t-L):(t-1),(t-L):(t-1)}^{-1} R_{(t-L):(t-1), t}
\end{aligned}
$$

The above result is based on a known result in statistics on conditional distributions derived from multivariate normal distribution [Anderson 1984].

The product of the above conditional multivariate normal distributions is a multivariate normal that reproduces exact detector correlations across time periods up to lag $L$ and approximates detector correlations just

Digital Threats: Research and Practice, Vol. 3, No. 1, Article 4. Publication date: October 2021. 
beyond lag $L$. Thus, we can use the resulting distribution to construct a Gaussian copula that reasonably approximates detector dependency when feature vectors are constructed using adjacent time periods.

The conditional distribution above is equivalent to the multivariate regression of $Z_{t}$ on $Z_{(t-L):(t-1)}$ :

$$
Z_{t}=\beta_{t,(t-L):(t-1)} z_{(t-L):(t-1)}+E_{t}
$$

where

$$
\begin{aligned}
\beta_{t \mid(t-L):(t-1)} & =R_{t,(t-L):(t-1)} R_{(t-L):(t-1),(t-L):(t-1)}^{-1} \\
E_{t} & \sim N\left(0, \Sigma_{t \mid(t-L):(t-1)}\right)
\end{aligned}
$$

This formulation makes the linear relationship (autocorrelation) between $Z_{t}$ and preceding random vectors $Z_{t-L}, \ldots, Z_{t-1}$ more apparent. Note that the residual variance matrix $R_{t \mid(t-L):(t-1)}$ equals the unconditional variance of $Z_{t}$ less the variance explained through its linear relationship with preceding random vectors.

Sequential Gaussian Copula Case 1: Known Correlation Matrix. Next, we construct the Gaussian copula algorithm for generating detector values one time period at a time. We sequentially apply the conditional multivariate normal distribution, obtaining at each time period: (1) correlated standard normal random values; (2) Gaussian copula uniform random values; and (3) correlated detector random values. For clarity we initially assume that the full $T p \times T p$ correlation matrix $R$ is completely known and positive definite.

To streamline the algorithm, prepend $L$ independent standard multivariate normal random vectors of dimension $p$, each of which is indepedent of the $T$ original normal random normal vectors. We then work with the $(L+T) p$ concatenated random vector $\left(Z_{1}, \ldots, Z_{L}, Z_{L+1}, \ldots, Z_{L+T}\right)$, which is distributed as multivariate normal with zero mean and covariance given by the $(L+T) p \times(L+T) p$ augmented correlation matrix

$$
R^{*}=\left(\begin{array}{cc}
I_{L p \times L p} & 0_{L P \times T p} \\
0_{T p \times L p} & R
\end{array}\right)
$$

where $R$ is the original $T p \times T p$ correlation matrix. Note that concatenation shifted the original time index forward by $L$ lags. In the algorithm below $s$ denotes the shifted time index while $t$ denotes the original time index.

The following are the key steps in the Gaussian copula algorithm for generating correlated random detector values.

Step 1: Do Steps 1.1 and 1.2 for $s=L+1, \ldots, L+T$. This loop computes multivariate regression parameters for all time periods using the augmented correlation matrix $R^{*}$.

Step 1.1: Compute the $p \times L p$ regression coefficient matrix at time $s$

$$
\beta_{s \mid(s-L):(s-1)}=R_{s,(s-L):(s-1)}^{*} R_{(s-L):(s-1),(s-L):(s-1)}^{*-1}
$$

Step 1.2: Compute $L p \times L p$ residual covariance matrix at time $s$

$$
\Sigma_{s \mid(s-L):(s-1)}^{*}=R_{s s}^{*}-R_{s,(s-L):(s-1)}^{*} R_{(s-L):(s-1),(s-L):(s-1)}^{*-1} R_{(s-L):(s-1), s}^{*}
$$

Step 2: For each individual do Step 2.1 and loop in Step 2.2

Step 2.1: Generate $L$ independent $p \times 1$ random vectors from $N\left(0, I_{p \times p}\right)$. Assign these vectors to $z_{1}, \ldots, z_{L}$.

Step 2.2 Do Step 2.2.1 to Step 2.2.4 for $s=L+1, \ldots, L+T$. At the end of this loop we have a complete vector of detector values over $T$ time periods for one individual.

Step 2.2.1: Generate a $p \times 1$ random vector from $N\left(0, \Sigma_{s \mid(s-L):(s-1)}^{*}\right)$. Assign these values to the residual vector $e_{s}$.

Step 2.2.2: Compute the $p \times 1$ multivariate normal random vector for time $s$ using regression coefficient matrix and residual vector at time $s$ and the normal random vectors realized in the preceding $L$ time periods.

$$
z_{s}=\beta_{s \mid(s-L):(s-1)} z_{(s-1):(s-L)}+e_{s}
$$

Digital Threats: Research and Practice, Vol. 3, No. 1, Article 4. Publication date: October 2021. 
Step 2.2.3: Transform elements of $z_{s}$ to copula uniforms to obtain $u_{s}=\left(u_{s 1}, \ldots, u_{s p}\right)^{\prime}$. (Note that marginals of $Z_{s}$ are standard normals.)

$$
u_{s i}=\Phi\left(z_{s i}\right), i=1, \ldots, p
$$

Step 2.2.4: Transform elements of $u_{s}$ to detector values using detector quantile functions at (original) time $t=s-L$. This step produces the $p \times 1$ detector vector realization $x_{t}$.

$$
x_{t i}=F_{X_{t, i}}^{-1}\left(u_{s i}\right), i=1, \ldots, p
$$

Following are some observations about the algorithm. First, in Steps 1.1 and 1.2 we only need detector correlations at the current and $L$ preceding time periods to compute multivariate regression parameters, so there is no need to approximate the full $T p \times T p$ correlation matrix, which would be challenging as number of time periods increases. Second, the algorithm can be generalized to T Copula by replacing the standard uniform random variable transformation in Step 2.2 .3 by

$$
u_{s i}=F_{t, v}\left(z_{s i} \sqrt{v / w}\right), i=1, \ldots, p
$$

where $F_{t, v}$ is the cumulative distribution of function of the $t$ distribution with $d f=v$ and $w$ is distributed as $\chi_{v}^{2}$.

Sequential Gaussian Copula Case 2: Approximate Correlation Matrix. As noted above, at given time iteration $s$, we only need the $L p \times L p$ submatrix $R_{(s-L): s,(s-L): s}^{*}$. Our approach for the case where the correlation matrix is approximated from provided correlations is to approximate $R_{(s-L): s,(s-L): s}^{*}$ separately at each iteration $s$. Because each iteration involves a positive definite transformation, correlations corresponding to the same detectors and time periods will differ across iterations. Our objective is to carry out approximations across iterations consistently so that these differences are as small as possible.

Below is the expanded version of Step 1 of the sequential Gaussian copula used in Case 1. At each step the expanded algorithm computes the provisional correlation matrix using the preceding step's final correlation matrix. The provisional correlation matrix is then transformed to be positive definite. The provisional and final correlation matrices at iteration $s$, denoted by $\tilde{R}^{*(S)}$ and $\widetilde{\widetilde{R}}^{*(S)}$, respectively, have dimension $(L+1) p \times(L+1) p$ and only includes time periods $s-L$ to $s$. Superscript “*” means matrix time indices are shifted $L$ lags forward, while superscipt " $(s)$ " means the matrix computed at time $s$.

Step 1: For $s=L+1, \ldots, L+T$ do Steps 1.1 to 1.4. This loop computes multivariate regression parameters for all time periods using approximate correlations.

Step 1.1: To get the algorithm started set the final correlation matrix at $s=L$ using

$$
\widetilde{\widetilde{R}}_{(1: L),(1: L)}^{*(L)}=I_{L p \times L p}
$$

Note this is just $R_{(1: L),(1: L)}^{*}$, the correlation matrix of prepended $L p$ independent standard normal random variables.

Step 1.2: For $s=L+1, \ldots, L+T$ do Steps 1.2.1 to 1.2.4. This loop approximates all correlations needed to compute all regression coefficient matrices and covariance matrices.

Step 1.2.1: Set provisional approximate correlations between time periods $s-L, \ldots, s-1$ at iteration $s$ using final correlations from iteration $s-1$.

$$
\tilde{R}_{(s-L):(s-1),(s-L):(s-1)}^{*(s)}=\widetilde{\widetilde{R}}_{(s-L):(s-1),(s-L):(s-1)}^{*(s-1)}
$$

Note that the LHS corresponds to the first $L$ periods included in $\tilde{R}^{*(S)}$ while the RHS corresponds to the last $L$ periods included in $\tilde{R}^{*(S-1)}$.

Digital Threats: Research and Practice, Vol. 3, No. 1, Article 4. Publication date: October 2021. 
Step 1.2.2: Set provisional correlations between time periods $s-L, \ldots, s-1$ and $s$ using an approximation method based on provided correlations. For $s^{\prime}=s-L, \ldots, s-1$ do:

$$
\begin{aligned}
& \tilde{R}_{s^{\prime}, s}^{*(s)}=\frac{1}{2} \operatorname{sgn}\left(R_{s^{\prime}, s}^{d}\right) \operatorname{abs}\left(R_{s^{\prime}, s}^{d}\right)^{\frac{1}{2}}\left(R_{s^{\prime}, s^{\prime}}+R_{s, s}\right) \operatorname{abs}\left(R_{s^{\prime}, s}^{d}\right)^{\frac{1}{2}} \operatorname{sgn}\left(R_{s^{\prime}, s}^{d}\right) \\
& \tilde{R}_{s^{\prime}, s}^{*(s)}=\left(\tilde{R}_{s^{\prime}, s}^{*(s)}\right)^{T}
\end{aligned}
$$

Step 1.2.3: Set provisional correlations at time $s$ using provided correlations.

$$
\tilde{R}_{s, s}^{*(s)}=R_{s, s}^{*}
$$

Step 1.2.4: Transform the provisional approximate correlation matrix to positive definite matrix, giving more weight to time $s-L, \ldots, s-1$. This step produces the final approximate correlations for iteration $s$.

$$
\widetilde{\widetilde{R}}_{(s-L): s,(s-L): s}^{*(s)}=\operatorname{PSD}\left(\tilde{R}_{(s-L): s,(s-L): s}^{*(s)}\right)
$$

Step 1.3: Compute the $p \times L p$ regression coefficient matrix at time $s$

$$
\beta_{s \mid(s-L):(s-1)}=\widetilde{\widetilde{R}}_{s,(s-L):(s-1)}^{*(s)} \stackrel{\approx}{R}_{(s-L):(s-1),(s-L):(s-1)}^{-1}
$$

Step 1.4: Compute $L p \times L p$ residual covariance matrix at time $s$

$$
\Sigma_{s \mid(s-L):(s-1)}^{*}=\widetilde{\widetilde{R}}_{s s}^{*(s)}-\widetilde{\widetilde{R}}_{s,(s-L):(s-1)}^{*(s)} \quad \widetilde{\widetilde{R}}_{(s-L):(s-1),(s-L):(s-1)}^{\tilde{R}^{-1}} \approx_{(s-L):(s-1), s}^{*(s)}
$$

At this point we have the multivariate regression parameters. To generate detector vector realizations for each individual, proceed to Step 2 of the preceding algorithm Gaussian copula algorithm for known correlation matrix.

In Step 1.2.4 the provisional correlations for time periods $s-L, \ldots, s-1$ (initialized in Step 1.2.1 using final values from the preceding iteration) were given more weight to force the final correlations for the same detectors and time periods to be as close as possible across iterations using the weighted positive definite transformation of [Higham 2002].

\section{REFERENCES}

Theodore W. Anderson. 1984. An Introduction to Multivariate Statistical Analysis (2nd ed.). Wiley, New York, NY.

Elise T. Axelrad, Paul J. Sticha, Oliver Brdiczka, and Jianqiang Shen. 2013. A Bayesian network model for predicting insider threats. In 2013 IEEE Security and Privacy Workshops, IEEE, San Francisco CA 82-89. https://doi.org/10.1109/SPW.2013.35

David P. Brown, Denis M. Buede, and Sean D. Vermillion. 2019. Improving insider threat detection through multi-modelling/data fusion. Procedia Computer Science 153 (2019), 100-107. https://doi.org/10.1016/j.procs.2019.05.060

Dennis M. Buede, Elise T. Axelrad, David P. Brown, Daniel W. Hudson, Kathryn B. Laskey, Paul J. Sticha, and Jordan L. Thomas. 2018. Inference enterprise models: An approach to organizational performance improvement. WIREs Data Mining Knowl Discov. 2018, e1277. https://doi.org/10.1002/widm.1277

Dawn M. Cappelli, Akash G. Desai, Andrew P. Moore, Timothy J. Shimeall, Elise A. Weaver, and Bradford J. Willke. 2006. Management and education of the risk of insider threat (MERIT): Mitigating the risk of sabotage to employers' information, systems, or networks. In Proceedings of the 24th International System Dynamics Conference. Nijmegen, Netherlands. https://proceedings.systemdynamics.org/2006/ proceed/papers/MOORE333.pdf.

Alvaro A. Cárdenas, Saurabh Amin, Zong-Syun Lin, Yu-Lun Huang, Chi-Yen Huang, and Shankar Sastry. 2011. Attacks against process control systems: risk assessment, detection, and response. In Proceedings of the 6th ACM Symposium on Information, Computer and Communications Security (ASIACCS '11). ACM, New York, NY, USA, 355-366. https://doi.org/10.1145/1966913.1966959

Welton Chang, Eva Chen, Barbara Mellers, and Philip Tetlock. 2016. Developing expert political judgment: The impact of training and practice on judgmental accuracy in geopolitical forecasting tournaments. Judgment and Decision Making 11, 5 (September 2016) 509-526.

Loren J. Chapman. 1967. Illusory correlation in observational report. Journal of Verbal Learning and Verbal Behavior 6, 1 (February 1967), 151-155. https://doi.org/10.1016/S0022-5371(67)80066-5

Allen I. Fleishman. 1978. A method for simulating non-normal distributions. Psychometrika 43 (1978), 521-532. https://doi.org/10.1007/ BF02293811

Gerd Gigerenzer. 2002. Calculated Risks: How to Know when Numbers Deceive You. Simon \& Schuster, New York, NY.

Digital Threats: Research and Practice, Vol. 3, No. 1, Article 4. Publication date: October 2021. 
Gerd Gigerenzer and Adrian Edwards. 2003. Simple tools for understanding risks: From innumeracy to insight. British Medical fournal 327, 741-744. https://doi.org/10.1136/bmj.327.7417.741

Frank L. Greitzer, Deborah A. Frincke, and Mariah. Zabriskie. 2011. Social/ethical issues in predictive insider threat monitoring. In: MJ Dark (Ed.), Information Assurance and Security Ethics in Complex Systems: Interdisciplinary Perspectives. Chapter 7, 132-161. IGI Global, Hershey, Pennsylvania. https://doi.org/10.4018/978-1-61692-245-0.ch007

Rodger W. Griffeth, Peter W. Hom, and Stefan Gaertner. 2000. A meta-analysis of antecedents and correlates of employee turnover: Update, moderator tests, and research implications for the next millennium. fournal of Management 26, 3, 463-488. https://doi.org/10.1016/S01492063(00)00043-X

Peter Hall and Susan R. Wilson. 1991. Two guidelines for bootstrap hypothesis testing. Biometrics 47, 2 (June 1991), 757-762. https://doi.org/ $10.2307 / 2532163$

Todd C. Hendrick. 2002. Fast fifth-order polynomial transforms for generating univariate and multivariate nonnormal distributions. Computational Statistics \& Data Analysis 40, 4 (October 2002), 685-711. https://doi.org/10.1016/S0167-9473(02)00072-5

Nicholas J. Higham. 2002. Computing the nearest correlation matrix - a problem from finance. IMA fournal of Numerical Analysis 22, 3 (July 2002), 329-343. https://doi.org/10.1093/imanum/22.3.329

Barbara A. Mellers and A. Peter McGraw. 1999. How to improve Bayesian reasoning: Comment on Gigerenzer and Hoffrage (1995). Psychological Review, 106, 2 (April 1999), 417-424. https://doi.org/10.1037/0033-295X.106.2.417

Andrew P. Moore, Dawn M. Cappelli, Thomas C. Caron, Eric Shaw, Derrick Spooner, and Randall F. Trzeciak. 2011. A preliminary model of insider theft of intellectual property. Fournal of Wireless Mobile Networks, Ubiquitous Computing, and Dependable Applications 2, 1 (March 2011), 28-49. https://doi.org/10.22667/JOWUA.2011.03.31.028

Michael Mount, Remus Ilies, and Erin Johnson. 2006. Relationship of personality traits and counterproductive work behaviors: The mediating effects of job satisfaction. Personnel Psychology 59, 3 (Autumn 2006), 591-622. https://doi.org/10.1111/j.1744-6570.2006.00048.x

Roger B. Nelsen. 2006. An Introduction to Copulas (2nd ed.). Springer, New York, NY.

Oscar L. Olvera Astivia and Bruno D. Zumbo. 2015. A cautionary note on the use of the Vale and Maurelli method to generate multivariate, nonnormal data for simulation purposes. Educ Psychol Meas. 75, 4 (August 2015), 541-567. https://doi.org/10.1177/0013164414548894

Fabian Pedregosa, Gaël Varoquaux, Alexandre Gramfort, Vincent Michel, Bertrand Thirion, Olivier Grisel, Mathieu Blondel, Peter Prettenhofer, Ron Weiss, Vincent Dubourg, Jake Vanderplas, Alexandre Passos, David Cournapeau, Matthieu Brucher, Matthieu Perrot, and Édouard Duchesnay. 2011. Scikit-learn: Machine learning in Python. Journal of Machine Learning Research 12, 85 (February 2011), 28252830.

Sandra L. Robinson and Rebecca J. Bennett. 1995. A typology of deviant workplace behaviors: a multidimensional scaling study. Academy of Management fournal 38, 2 (April 1995), 555-572. https://doi.org/10.2307/256693

John Ruscio and Walter Kaczetow. 2008. Simulating multivariate nonnormal data using an iterative algorithm. Multivariate Behavioral Research 43, 3 (July-Sep 2008), 335-381. https://doi.org/10.1080/00273170802285693

Abe Sklar. 1959. Fonctions de repartition an dimensions et leurs marges. Publ. inst. statist. univ. Paris 8, $229-231$.

Philip E. Tetlock and Dan Gardner. 2015. Superforecasting: The Art and Science of Prediction. Crown Publishers, New York, NY.

Amos Tversky and Daniel Kahneman. 1973. Availability: A heuristic for judging frequency and probability. Cognitive Psychology. 5, 2 (September 1973), 207-232. https://doi.org/10.1016/0010-0285(73)90033-9

C. David Vale and Vincent A. Maurelli. 1983. Simulating multivariate nonnormal distributions. Psychometrika 48, 3 (September 1983), 465-471. https://doi.org/10.1007/BF02293687

Bahram Yousefi, Mohanad Ajina, Muhammad Imran, and Kathryn Laskey. 2018. A non-parametric approach to simulate panel data. In Proceedings of the 2018 Winter Simulation Conference (WSC'18). IEEE Press, 515-524. https://doi.org/10.1109/WSC.2018.8632547

Received September 2020; revised March 2021; accepted March 2021

Digital Threats: Research and Practice, Vol. 3, No. 1, Article 4. Publication date: October 2021. 